\title{
The classification of left-invariant para-Kähler structures on simply connected four-dimensional Lie groups
}

https://doi.org/10.1515/coma-2021-0127

Received April 15, 2021; accepted December 17, 2021

\begin{abstract}
We give a complete classification of left invariant para-Kähler structures on four-dimensional simply connected Lie groups up to an automorphism. As an application we discuss some curvatures properties of the canonical connection associated to these structures as flat, Ricci flat and existence of Ricci solitons.
\end{abstract}

Keywords: Symplectic Lie algebras, para-Kähler structures, Ricci soliton

MSC: 53D05; 53C30; 53C25

\section{Introduction and main results}

An almost para-complex structure on a $2 n$-dimensional manifold $M$ is a field of endomorphisms $K: T M \rightarrow$ $T M$ such that $K^{2}=I d_{T M}$ and the two eigendistributions $T^{ \pm} M:=\operatorname{ker}(I d \pm K)$ have the same rank. An almost para-complex structure $K$ is said to be integrable if the distributions $T^{ \pm} M$ are involutive. This is equivalent to the vanishing of the Nijenhuis tensor $N_{K}$ defined by

$$
N_{K}(X, Y)=[X, Y]+[K X, K Y]-K[K X, Y]-K[X, K Y],
$$

for vector fields $X, Y$ on $M$. In such a case $K$ is called a para-complex structure. A para-Kähler structure on a manifold $M$ is a pair $(\langle.,\rangle, K$.$) where \langle.,$.$\rangle is a pseudo-Riemannian metric and K$ is a parallel skew-symmetric para-complex structure. If $(\langle.,\rangle, K$.$) is a para-Kähler structure on M$, then $\omega=\langle.,.\rangle \circ K$ is a symplectic structure and the \pm 1 -eigendistributions $T^{ \pm} M$ of $K$ are two integrable $\omega$-Lagrangian distributions. Due to this, a paraKähler structure can be identified with a bi-Lagrangian structure $\left(\omega, T^{ \pm} M\right)$ where $\omega$ is a symplectic structure and $T^{ \pm} M$ are two integrable Lagrangian distributions. Moreover the Levi-Civita connection associated to the neutral metric $\langle.,$.$\rangle coincides with the canonical connection associate to the bi-Lagrangian structure (the$ unique symplectic connection with parallelizes both foliations [9]). For a survey on paracomplex geometry see [6] and for background on bi-Lagrangian structures and their associated connections, the survey [7] is a good starting point and contains further references (See as well [1] and [5]).

Suppose now that $M$ is a Lie group $G$ and $\omega,\langle.,$.$\rangle and K$ are left invariant. If we denote by $\mathfrak{g}$ the Lie algebra of $G$, then $(\langle.,\rangle, K$.$) is determined by their restrictions to the Lie algebra \mathfrak{g}$. In this situation, $\left(\mathfrak{g},\langle., .\rangle_{e}, K_{e}\right)$ or $\left(\mathfrak{g}, \omega_{e}, K_{e}\right)$ is called a para-Kähler Lie algebra (e is unit of $G$ ). In the rest of this paper a para-Kähler Lie algebra will be noted $(\mathfrak{g}, \omega, K)$. Recall that two para-Kähler Lie algebras $\left(\mathfrak{g}_{1}, \omega_{1}, K_{1}\right)$ and $\left(\mathfrak{g}_{2}, \omega_{2}, K_{2}\right)$ are said to be equivalent if there exists an isomorphism of Lie algebras $T: \mathfrak{g}_{1} \rightarrow \mathfrak{g}_{2}$ such as $T^{\star} \omega_{2}=\omega_{1}$ and $T_{\star} K_{1}=K_{2}$. Para-Kähler (bi-Lagrangian) structures on Lie algebras in general have been studied, for example, in [2], [3] and [4]. In [8], there is a study of the existence of bi-Lagrangian structures on symplectic nilpotent Lie alge-

^Corresponding Author: M. W. Mansouri: Université Ibno Tofail, Faculté des Sciences. Kénitra-Maroc, E-mail: mansourimohammed.wadia@uit.ac.ma

A. Oufkou: Université Ibno Tofail, Faculté des Sciences. Kénitra-Maroc, E-mail: ahmed.oufkou@uit.ac.ma 
bras of dimension 2,4 and 6. A first classification of para-Kähler structures on four-dimensional Lie algebras was obtained by Calvaruso in [6]. Another description based on the classification of symplectic Lie algebras is proposed by Smolentsev and Shagabudinova in [12]. Benayadi and Boucetta provide in [3] a new characterization of para-Kähler Lie algebras using left symmetric bialgebras inroduced by Bai in [2]. Based on this characterization, we propose in this paper the classification of para-Kähler structures on four-dimensional Lie algebras. Notice that our classification is more complete and precise than the other classifications existing in the literature.

Notations: For $\left\{e_{1}, e_{2}, e_{3}, e_{4}\right\}$ a basis of $\mathfrak{g}$, we denote by $\left\{e^{1}, e^{2}, e^{3}, e^{4}\right\}$ the dual basis on $\mathfrak{g}^{\star}$ and $e^{i j}$ the two-form $e^{i} \wedge e^{j}, \dot{e}^{i j}$ is the symmetric two-form $e^{i} \odot e^{j}$ and $E_{i j}$ is the endomorphism which sends $e_{j}$ to $e_{i}$ and vanishes on $e_{k}$ for $k /=j$.

A para-Kähler Lie algebra $(\mathfrak{g},\langle.,\rangle, K$.$) is necessarily a symplectic Lie algebra (\mathfrak{g}, \omega)$. It is well known that a symplectic four-dimensional Lie algebra is necessarily solvable. The classification of symplectic fourdimensional Lie algebras $(\mathfrak{g}, \omega)$ is given by the following Table (see [10]).

Table 1: Symplectic four-dimensional Lie algebras

$\left(\mu \geq 0,-1 \leq \beta<1,-1<\alpha<0, \delta>0\right.$ and $\left.\lambda \geq \frac{1}{2}, \lambda=1,2\right)$.

\begin{tabular}{|c|c|c|}
\hline Case & No vanishing brackets & $\omega$ \\
\hline $\mathfrak{r h}_{3}$ & {$\left[e_{1}, e_{2}\right]=e_{3}$} & $e^{14}+e^{23}$ \\
\hline$\overline{\mathfrak{r r}_{3,0}}$ & {$\left[e_{1}, e_{2}\right]=e_{2}$} & $e^{12}+e^{34}$ \\
\hline $\mathfrak{r r}_{3,-1}$ & {$\left[e_{1}, e_{2}\right]=e_{2},\left[e_{1}, e_{3}\right]=-e_{3}$} & $e^{14}+e^{23}$ \\
\hline$\overline{\mathfrak{r r r}_{3,0}^{\prime}}$ & {$\left[e_{1}, e_{2}\right]=-e_{3},\left[e_{1}, e_{3}\right]=e_{2}$} & $e^{14}+e^{23}$ \\
\hline $\mathfrak{r}_{2} \mathfrak{r}_{2}$ & {$\left[e_{1}, e_{2}\right]=e_{2},\left[e_{3}, e_{4}\right]=e_{4}$} & $e^{12}+\mu e^{13}+e^{34}$ \\
\hline $\mathfrak{r}_{2}^{\prime}$ & $\begin{array}{l}{\left[e_{1}, e_{3}\right]=e_{3},\left[e_{1}, e_{4}\right]=e_{4},} \\
{\left[e_{2}, e_{3}\right]=e_{4},\left[e_{2}, e_{4}\right]=-e_{3}}\end{array}$ & $e^{14}+e^{23}$ \\
\hline $\mathfrak{n}_{4}$ & {$\left[e_{4}, e_{1}\right]=e_{2},\left[e_{4}, e_{2}\right]=e_{3}$} & $e^{12}+e^{34}$ \\
\hline $\mathfrak{r}_{4,0}$ & {$\left[e_{4}, e_{1}\right]=e_{1},\left[e_{4}, e_{3}\right]=e_{2}$} & $e^{14} \mp e^{23}$ \\
\hline $\mathfrak{r}_{4,-1}$ & {$\left[e_{4}, e_{1}\right]=e_{1},\left[e_{4}, e_{2}\right]=-e_{2},\left[e_{4}, e_{3}\right]=e_{2}-e_{3}$} & $e^{13}+e^{24}$ \\
\hline $\mathfrak{r}_{4,-1, \beta}$ & {$\left[e_{4}, e_{1}\right]=e_{1},\left[e_{4}, e_{2}\right]=-e_{2},\left[e_{4}, e_{3}\right]=\beta e_{3}$} & $e^{12}+e^{34}$ \\
\hline $\mathfrak{r}_{4, \alpha,-\alpha}$ & {$\left[e_{4}, e_{1}\right]=e_{1},\left[e_{4}, e_{2}\right]=\alpha e_{2},\left[e_{4}, e_{3}\right]=-\alpha e_{3}$} & $e^{14}+e^{23}$ \\
\hline$\overline{\mathfrak{r}_{4,0, \delta}^{\prime}}$ & {$\left[e_{4}, e_{1}\right]=e_{1},\left[e_{4}, e_{2}\right]=-\delta e_{3},\left[e_{4}, e_{3}\right]=\delta e_{2}$} & $e^{14} \mp e^{23}$ \\
\hline $\mathfrak{d}_{4,1}$ & $\begin{array}{l}{\left[e_{1}, e_{2}\right]=e_{3},\left[e_{4}, e_{3}\right]=e_{3},} \\
{\left[e_{4}, e_{1}\right]=e_{1}}\end{array}$ & $\begin{array}{c}e^{12}-e^{34} \\
e^{12}-e^{34}+e^{24}\end{array}$ \\
\hline $\mathfrak{d}_{4,2}$ & $\begin{array}{l}{\left[e_{1}, e_{2}\right]=e_{3},\left[e_{4}, e_{3}\right]=e_{3},} \\
{\left[e_{4}, e_{1}\right]=2 e_{1},\left[e_{4}, e_{2}\right]=-e_{2}}\end{array}$ & $\begin{array}{l}e^{12}-e^{34} \\
e^{14} \mp e^{23}\end{array}$ \\
\hline $\mathfrak{d}_{4, \lambda}$ & $\begin{array}{l}{\left[e_{1}, e_{2}\right]=e_{3},\left[e_{4}, e_{3}\right]=e_{3},} \\
{\left[e_{4}, e_{1}\right]=\lambda e_{1},\left[e_{4}, e_{2}\right]=(1-\lambda) e_{2}}\end{array}$ & $e^{12}-e^{34}$ \\
\hline $\mathfrak{d}_{4, \delta}^{\prime}$ & $\begin{array}{l}{\left[e_{1}, e_{2}\right]=e_{3},\left[e_{4}, e_{1}\right]=\frac{\delta}{2} e_{1}-e_{2}} \\
{\left[e_{4}, e_{3}\right]=\delta e_{3},\left[e_{4}, e_{2}\right]=e_{1}+\frac{\delta}{2} e_{2}}\end{array}$ & $\mp\left(e^{12}-\delta e^{34}\right)$ \\
\hline $\mathfrak{h}_{4}$ & $\begin{array}{l}{\left[e_{1}, e_{2}\right]=e_{3},\left[e_{4}, e_{3}\right]=e_{3},} \\
{\left[e_{4}, e_{1}\right]=\frac{1}{2} e_{1},\left[e_{4}, e_{2}\right]=e_{1}+\frac{1}{2} e_{2}}\end{array}$ & $\mp\left(e^{12}-e^{34}\right)$ \\
\hline
\end{tabular}

Our main result is the following.

Theorem 1.1. Let $(\mathfrak{g}, \omega, K)$ be a four-dimensional para-Kähler Lie algebra and $x \in \mathbb{R}$. Then $(\mathfrak{g}, \omega, K)$ is isomorphic to one of the following Lie algebras with the given para-Kähler structures:

Lie algebra $\mathfrak{r h}_{3}$

$$
\begin{aligned}
& \text { For } \omega=e^{14}+e^{23} \\
& \qquad K_{1}= \pm\left(E_{11}-E_{22}+E_{33}-E_{44}\right)
\end{aligned}
$$




$$
K_{2}= \pm\left(-E_{11}+E_{21}+E_{22}-E_{33}-E_{43}+E_{44}\right)
$$

\section{Lie algebra $\mathfrak{r r}_{3,0}$}

$$
\text { For } \begin{aligned}
\omega & =e^{12}+e^{34} \\
K_{1} & = \pm\left(-E_{11}+E_{22}-E_{33}+E_{44}\right) \\
K_{2} & =E_{11}+x E_{12}-E_{22}+E_{33}-E_{44}, x \neq 0
\end{aligned}
$$

\section{Lie algebra $\mathfrak{r r}_{3,-1}$}

$$
\text { For } \begin{aligned}
\omega & =e^{14}+e^{23} \\
K_{1} & = \pm\left(-E_{11}+E_{22}-E_{33}+E_{44}\right) \\
K_{2} & = \pm\left(E_{11}+E_{22} \pm E_{23}-E_{33}-E_{44}\right) \\
K_{3} & =E_{11}+E_{22}+x E_{14}-E_{33}-E_{44}, x \neq 0 \\
K_{3} & =-E_{11}+E_{22} \pm E_{32}-E_{33}+E_{44}
\end{aligned}
$$

\section{Lie algebra $\mathfrak{r}_{2} \mathfrak{r}_{2}$}

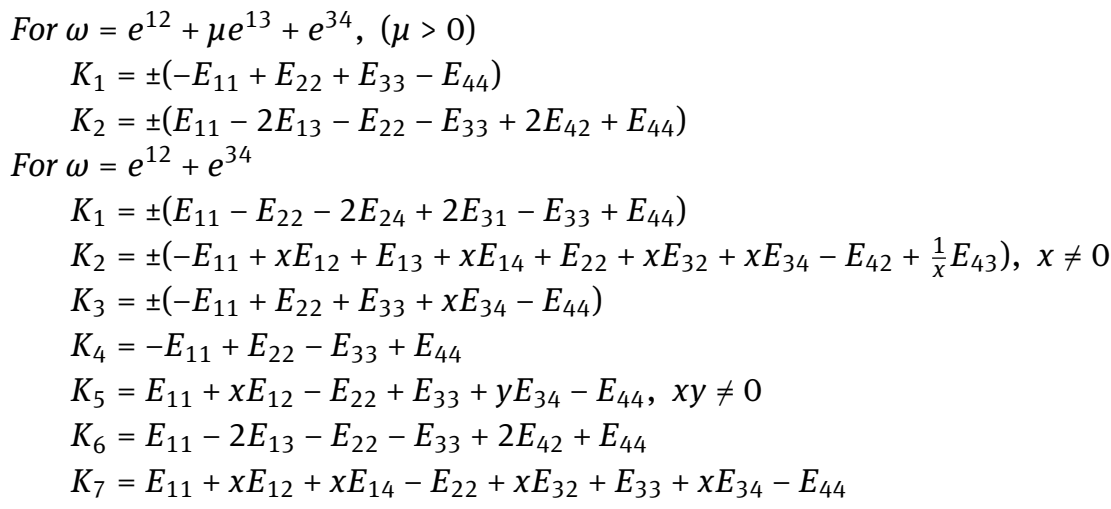

\section{Lie algebra $\mathrm{r}_{2}^{\prime}$}

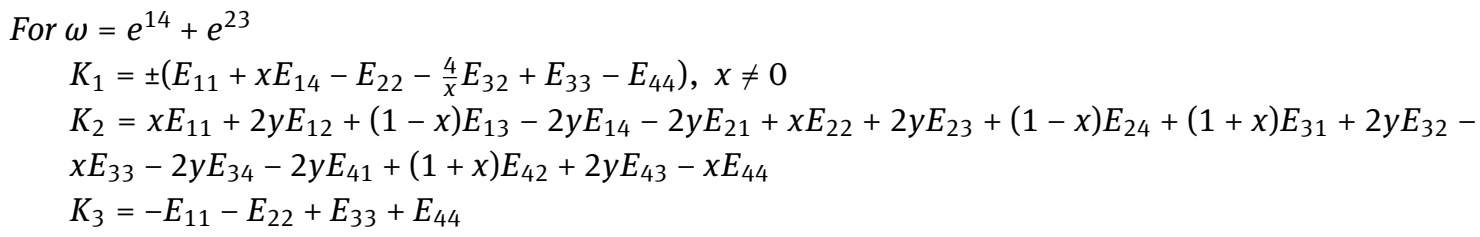

\section{Lie algebra $\mathfrak{r}_{4,0}$}

$$
\text { For } \begin{aligned}
\omega & =e^{14} \pm e^{23} \\
K & = \pm\left(E_{11}-E_{22}+E_{33}-E_{44}\right)
\end{aligned}
$$

\section{Lie algebra $\mathfrak{r}_{4,-1}$}

$$
\begin{aligned}
& \text { For } \omega=e^{13}+e^{24} \\
& \qquad K_{1}= \pm\left(E_{11}+x E_{13}-E_{22}-E_{33}+E_{44}\right)
\end{aligned}
$$

Lie algebra $\mathfrak{r}_{4,-1, \beta}(-1<\beta<1)$

$$
\text { For } \begin{aligned}
\omega & =e^{12}+e^{34} \\
& K_{1}= \pm\left(E_{11} \pm E_{12}-E_{22}-E_{33}+E_{44}\right) \\
K_{2} & = \pm\left(E_{11}-E_{22}-E_{33}+E_{44}\right)
\end{aligned}
$$




$$
\begin{aligned}
& K_{3}= \pm\left(E_{11}-E_{22}+x E_{34}+\frac{1}{x} E_{43}\right), x \neq 0 \\
& K_{4}= \pm\left(E_{11}-E_{22}+E_{33}-E_{44}\right)
\end{aligned}
$$

\section{Lie algebra $\mathfrak{r}_{4,-1,-1}$}

$$
\text { For } \begin{aligned}
\omega= & e^{12}+e^{34} \\
& K_{1}= \pm\left(-E_{11} \pm E_{21}+E_{22}-E_{33}+E_{44}\right) \\
K_{2} & = \pm\left(-E_{11}+E_{22}-E_{33}+x E_{43}+E_{44}\right) \\
K_{3} & = \pm\left(E_{11} \pm E_{12}-E_{22}-E_{33}+E_{44}\right) \\
K_{4} & = \pm\left(E_{11}-E_{22}-E_{33}+x E_{43}+E_{44}\right) \\
K_{5} & =-E_{11}+\chi E_{21}+E_{22}+E_{23}-E_{33}+E_{41}+E_{44}
\end{aligned}
$$

Lie algebra $\mathfrak{r}_{4, \alpha,-\alpha}(-1<\alpha<0)$

$$
\text { For } \begin{aligned}
& \omega= e^{14}+e^{23} \\
& K_{1}= \pm\left(-E_{11}+E_{22} \pm E_{23}-E_{33}+E_{44}\right) \\
& K_{2}= \pm\left(-E_{11}+E_{22}-E_{33}+x E_{41}+E_{44}\right) \\
& K_{3}= \pm\left(E_{11}+E_{22}-E_{33}-E_{44}\right) \\
& K_{4}= \pm\left(E_{11}+E_{22} \pm E_{32}-E_{33}-E_{44}\right)
\end{aligned}
$$

\section{Lie algebra $\mathfrak{d}_{4,1}$}

$$
\begin{aligned}
& \text { For } \omega=e^{12}-e^{34} \\
& K_{1}= \pm\left(E_{11} \pm E_{12}-E_{22}-E_{33}+E_{44}\right) \\
& K_{2}= \pm\left(E_{11}-E_{22}-E_{33}+x E_{43}+E_{44}\right) \\
& K_{3}= \pm\left(-E_{11}+E_{22}-E_{33}+x E_{43}+E_{44}\right) \\
& K_{4}= \pm\left(E_{11}+E_{21}-E_{22}+x E_{23}+E_{33}-x E_{41}-E_{44}\right) \\
& K_{5}= \pm\left(-E_{11} \pm E_{21}+E_{22}-E_{33}+E_{44}\right) \\
& \text { For } \omega=e^{12}-e^{34}+e^{24} \\
& \\
& K_{1}= \pm\left(E_{11}+x E_{12}-E_{22}-E_{33}+E_{44}\right)
\end{aligned}
$$

\section{Lie algebra $\mathfrak{d}_{4,2}$}

$$
\begin{aligned}
& \text { For } \omega=e^{12}-e^{34} \\
& \qquad \begin{aligned}
K_{1}= \pm\left(E_{11}+x E_{12}-E_{22}-E_{33}+E_{44}\right) \\
K_{2}= \pm\left(E_{11}-E_{22}+E_{33}+x E_{43}-E_{44}\right)
\end{aligned} \\
& \text { For } \omega=e^{14}-e^{23} \\
& \quad K_{1}= \pm\left(E_{11}-E_{22}+E_{33}+x E_{41}-E_{44}\right) \\
& K_{2}= \pm\left(-E_{11}-E_{22}+2 E_{31}+x E_{32}+E_{33}-2 E_{42}+E_{44}\right) \\
& K_{3}= \pm\left(-E_{11}+E_{22}+x E_{21}+x E_{23}-E_{33}+x E_{41}+x E_{43}+E_{44}\right) \\
& K_{4}= \pm\left(E_{11}-E_{22}+x E_{21}+x E_{23}+E_{33}+x E_{41}+x E_{43}-E_{44}\right) \\
& K_{5}=E_{11}-E_{22}-x E_{23}+E_{33}+\frac{x}{2} E_{41}-E_{44}, x \neq 0 \\
& \text { For } \begin{aligned}
\omega & =e^{14}+e^{23} \\
K_{1} & = \pm\left(E_{11}-E_{22}+E_{33}+x E_{41}-E_{44}\right) \\
K_{2} & =E_{11}-E_{22}+x E_{23}+E_{33}-\frac{x}{2} E_{41}-E_{44}, x \neq 0
\end{aligned}
\end{aligned}
$$

\section{Lie algebra $\mathfrak{d}_{4, \frac{1}{2}}$}

$$
\begin{aligned}
& \text { For } \omega=e^{12}-e^{34} \\
& \qquad \begin{array}{l}
K_{1}=E_{11}-E_{22}-E_{33}+x E_{43}+E_{44}, x \neq 0 \\
K_{2}= \pm\left(E_{11}-E_{22}+E_{33}-E_{44}\right)
\end{array}
\end{aligned}
$$


Lie algebra $\mathfrak{d}_{4, \lambda}\left(\lambda>\frac{1}{2}, \lambda=1,2\right)$

$$
\text { For } \begin{aligned}
\omega & =e^{12}-e^{34} \\
K_{1} & = \pm\left(E_{11} \pm E_{12}-E_{22}-E_{33}+E_{44}\right) \\
K_{2} & = \pm\left(-E_{11} \pm E_{21}+E_{22}-E_{33}+E_{44}\right) \\
K_{3} & = \pm\left(E_{11}-E_{22}-E_{33}+x E_{43}+E_{44}\right) \\
K_{4} & =-E_{11}+E_{22}-E_{33}+x E_{43}+E_{44} \\
K_{5} & =E_{11}+x E_{21}-E_{22}+E_{33}-E_{44}
\end{aligned}
$$

\section{Lie algebra $\mathfrak{h}_{4}$}

$$
\begin{aligned}
& \text { For } \omega= \pm\left(e^{12}-e^{34}\right) \\
& K_{1}= \pm\left(E_{11}-E_{22}-E_{33}+E_{44}\right)
\end{aligned}
$$

Corollary 1.1. The symplectic Lie algebras $\mathfrak{r r}_{3,0}^{\prime}, \mathfrak{n}_{4}, \mathfrak{r}_{4,0, \delta}^{\prime}$ and $\mathfrak{d}_{4, \delta}^{\prime}$ do not admit a para-Kähler structure.

The paper is organized as follows. Section 2 contains the basic results which are essential to the classification of four-dimensional para-Kähler Lie algebras (proof of the Theorem 1.1). Theorem 2.1 and Theorem 2.2 are the key steps in this proof. Section 3 is devoted to some curvature properties of four-dimensional paraKähler metrics. Section 4 contains the tables of Theorem 2.2 and the isomorphisms tables used in the proof of Theorem 1.1.

The software Maple $18^{\circledR}$ has been used to check all needed calculations.

\section{Proof of Theorem 1.1}

In this section we begin with a reminder of the new approach introduced by Benayadi and Boucetta in [3], which characterizes the para-Kähler Lie algebras.

Recall that, a para-Kähler Lie algebra $(\mathfrak{g},\langle.,\rangle, K$.$) carries a Levi-Civita product witch is characterized by$ Koszul's formula:

$$
2\langle u . v, w\rangle=\langle[u, v], w\rangle+\langle[w, u], v\rangle+\langle[w, v], u\rangle .
$$

The subalgebras $\mathfrak{g}^{1}=\operatorname{ker}\left(K-I d_{\mathfrak{g}}\right)$ and $\mathfrak{g}^{-1}=\operatorname{ker}\left(K+I d_{\mathfrak{g}}\right)$ have the following properties, $\mathfrak{g}^{1}$ and $\mathfrak{g}^{-1}$ are isotropic with respect to $\langle.$, . . $\rangle$, Lagrangian with respect to $\omega$ and checking that $\mathfrak{g}=\mathfrak{g}^{1} \oplus \mathfrak{g}^{-1}$, moreover the restriction of the Levi-Civita product on $\mathfrak{g}^{1}$ and $\mathfrak{g}^{-1}$ induces a left symmetric structure. i.e. for any $u, v, w \in \mathfrak{g}^{1}$ (resp. $\mathfrak{g}^{-1}$ ),

$$
\operatorname{ass}(u, v, w)=\operatorname{ass}(v, u, w)
$$

where $\operatorname{ass}(u, v, w)=(u . v) . w-u .(v . w)$. In particular, $\mathfrak{g}^{1}$ and $\mathfrak{g}^{-1}$ are left symmetric algebras.

For any $u \in \mathfrak{g}^{-1}$, let $u^{\star}$ denote the element of $\left(\mathfrak{g}^{1}\right)^{\star}$ given by $u^{\star}(v)=\langle u, v\rangle$. The map $u \longmapsto u^{\star}$ realizes an isomorphism between $\mathfrak{g}^{-1}$ and $\left(\mathfrak{g}^{1}\right)^{\star}$. Thus, we can identify $(\mathfrak{g},\langle.,\rangle, K$.$) relative to the phase space \left(\mathfrak{g}^{1} \oplus\right.$ $\left.\left(\mathfrak{g}^{1}\right)^{\star},\langle., .\rangle_{0}, K_{0}\right)$, where $\langle., .\rangle_{0}$ and $K_{0}$ are given by:

$$
\langle u+\alpha, v+\beta\rangle_{0}=\alpha(v)+\beta(u) \text { and } K_{0}(u+\alpha)=u-\alpha .
$$

Both $\mathfrak{g}^{1}$ and $\left(\mathfrak{g}^{1}\right)^{\star}$ carry a left symmetric algebra structure. For any $u \in \mathfrak{g}^{1}$ and for any $\alpha \in\left(\mathfrak{g}^{1}\right)^{\star}$, we denote $L_{u}: \mathfrak{g}^{1} \rightarrow \mathfrak{g}^{1}$ and $L_{\alpha}:\left(\mathfrak{g}^{1}\right)^{\star} \rightarrow\left(\mathfrak{g}^{1}\right)^{\star}$ as the left multiplication by $u$ and $\alpha$, respectively, i.e., for any $v \in \mathfrak{g}^{1}$ and any $\beta \in\left(\mathfrak{g}^{1}\right)^{\star}$,

$$
L_{u} v=u . v \quad \text { and } \quad L_{\alpha} \beta=\alpha . \beta .
$$

The Levi-Civita product and the Lie bracket on $\mathfrak{g}$ are determined entirely by their restrictions to $\left(\mathfrak{g}^{1}\right)^{\star}$ and $\mathfrak{g}^{1}:$ For any $u \in \mathfrak{g}^{1}$ and for any $\alpha \in\left(\mathfrak{g}^{1}\right)^{\star}$,

$$
u . \alpha=L_{u}^{t} \alpha \quad \text { and } \quad \alpha . u=-L_{\alpha}^{t} u .
$$


Conversely, let $U$ be a finite dimensional vector space and $U^{\star}$ is its dual space. We suppose that both $U$ and $U^{\star}$ have the structure of a left symmetric algebra. We extend the products on $U$ and $U^{\star}$ to $U \oplus U^{\star}$ for any $X, Y \in U$ and for any $\alpha, \beta \in U^{\star}$, by putting

$$
(X+\alpha) \cdot(Y+\beta)=X . Y-L_{\alpha}^{t} Y-L_{X}^{t} \beta+\alpha . \beta .
$$

We say that two left symmetric products on $U$ and $U^{\star}$ are Lie-extendible if the commutator of the product on $U \oplus U^{\star}$ given by (1) is a Lie bracket. In this case we have the following theorem:

Theorem 2.1. [3] Let $(U,$.$) and \left(U^{\star},.\right)$ be two Lie-extendible left symmetric products. Then, $\left(U \oplus U^{\star},\langle., .\rangle_{0}, K_{0}\right)$, endowed with the Lie algebra bracket associated with the product given by (1) is a para-Kähler Lie algebra. Where $\omega_{0},\langle., .\rangle_{0}$ and $K_{0}$ are given by:

$$
\omega_{0}(u+\alpha, v+\beta)=\beta(u)-\alpha(v), \quad\langle u+\alpha, v+\beta\rangle_{0}=\alpha(v)+\beta(u) \quad \text { and } \quad K_{0}(u+\alpha)=u-\alpha .
$$

Moreover, all para-Kähler Lie algebras are obtained in this manner.

Remark 1. In the construction of the paracomplex structure associated to the bi-Lagrangian structure we have the choice between $\left(\omega, K_{0}\right)$ and $\left(\omega,-K_{0}\right)$ which are not necessarily isomorphic. We take that into consideration by giving all the para-kähler structures up to isomorphism.

Now let $U$ be a 2-dimensional vector space and $U^{*}$ its dual space and let $\left\{e_{1}, e_{2}\right\},\left\{e_{3}, e_{4}\right\}$ be a basis of $U$ and $U^{\star}$. We base on the previous theorem and the classification of real left-symmetric algebras in dimension 2 listed below (see Theorem 1.2. of [11])

$$
\begin{array}{l|l}
\mathfrak{b}_{1, \alpha}: e_{2} \cdot e_{1}=e_{1}, e_{2} \cdot e_{2}=\alpha \cdot e_{2} & \mathfrak{b}_{4}: e_{1} \cdot e_{2}=e_{1}, e_{2} \cdot e_{2}=e_{1}+e_{2} \\
\mathfrak{b}_{2}: e_{2} \cdot e_{1}=e_{1}, e_{2} \cdot e_{2}=e_{1}+e_{2} & \mathfrak{b}_{5}^{+}: e_{1} \cdot e_{1}=e_{2}, e_{2} \cdot e_{1}=-e_{1}, e_{2} \cdot e_{2}=-2 e_{2} \\
\mathfrak{b}_{3, \alpha} \alpha=0: e_{1} \cdot e_{2}=e_{1}, e_{2} \cdot e_{1}=\left(1-\frac{1}{\alpha}\right) e_{1}, e_{2} \cdot e_{2}=e_{2} & \mathfrak{b}_{5}^{-}: e_{1} \cdot e_{1}=-e_{2}, e_{2} \cdot e_{1}=-e_{1}, e_{2} \cdot e_{2}=-2 e_{2} \\
& \\
\mathfrak{c}_{1}: \text { Trivial left-symmetric algebra } & \mathfrak{c}_{2}: e_{2} \cdot e_{2}=e_{2} \\
\mathfrak{c}_{5}^{+}: e_{2} \cdot e_{2}=e_{2}, e_{2} \cdot e_{1}=e_{1}, e_{1} \cdot e_{2}=e_{1}, e_{1} \cdot e_{1}=e_{2} & \mathfrak{c}_{3}: e_{2} \cdot e_{2}=e_{1} \\
\mathfrak{c}_{5}^{-}: e_{2} \cdot e_{2}=e_{2}, e_{2} \cdot e_{1}=e_{1}, e_{1} \cdot e_{2}=e_{1}, e_{1} \cdot e_{1}=-e_{2} & \mathfrak{c}_{4}: e_{2} \cdot e_{2}=e_{2}, e_{2} \cdot e_{1}=e_{1}, e_{1} e_{2}=e_{1} \cdot
\end{array}
$$

Remark 2. $\mathfrak{b}$ stands for algebras with non-commutative associated Lie algebra and $\mathfrak{c}$ stands for algebras with commutative associated Lie algebra .

Theorem 2.2. Let $(\mathfrak{g},\langle.,\rangle, K$.$) be a four-dimensional para-Kähler Lie algebra. Then there exists a basis$ $\left\{e_{1}, e_{2}, e_{3}, e_{4}\right\}$ of $\mathfrak{g}$ such that

$$
\omega=e^{13}+e^{24}, \quad\langle., .\rangle=\dot{e}^{13}+\dot{e}^{24} \text { and } K=E_{11}+E_{22}-E_{33}-E_{44}
$$

and the non vanishing Lie brackets as listed in the Table 4 and 5.

Proof. We will give the proof in the case $\mathcal{B}_{2}$ since all cases should be handled in a similar way. In that case the left-symmetric product in $U$ is given by $e_{2} \cdot e_{1}=e_{1}, e_{2} \cdot e_{2}=e_{1}+e_{2}$ and let

$$
\begin{aligned}
& e_{3} \cdot e_{3}=a_{33} e_{3}+b_{33} e_{4} \\
& e_{3} \cdot e_{4}=a_{34} e_{3}+b_{34} e_{4} \\
& e_{4} \cdot e_{3}=a_{43} e_{3}+b_{43} e_{4} \\
& e_{4} \cdot e_{4}=a_{44} e_{3}+b_{44} e_{4}
\end{aligned}
$$

be an arbitrary product in $U^{\star}$, let's look for products in $U^{\star}$ which satisfy the Jacobi identity $\oint\left[\left[e_{i}, e_{j}\right], e_{k}\right]=0$ with $1 \leq i<j<k \leq 4$, where $\oint$ denotes the cyclic sum. 
The identities $\oint\left[\left[e_{1}, e_{2}\right], e_{3}\right]=0$ and $\oint\left[\left[e_{1}, e_{2}\right], e_{4}\right]=0$ are equivalent to

$$
\left\{\begin{array}{l}
b_{34}+a_{33}+a_{43}=0 \\
a_{44}=0 \\
b_{44}+a_{43}=0 .
\end{array}\right.
$$

Suppose that $a_{44}=0, b_{44}+a_{43}=0$ and $b_{34}=-a_{33}-a_{43}$, the identities $\oint\left[\left[e_{1}, e_{3}\right], e_{4}\right]=0$ and $\oint\left[\left[e_{2}, e_{3}\right], e_{4}\right]=0$ are equivalent to

$$
\left\{\begin{array} { l } 
{ a _ { 3 3 } a _ { 3 4 } - 2 a _ { 3 3 } a _ { 4 3 } - a _ { 3 4 } b _ { 4 3 } - a _ { 4 3 } ^ { 2 } - a _ { 4 3 } b _ { 4 3 } = 0 } \\
{ a _ { 3 4 } ( a _ { 3 4 } + a _ { 4 3 } ) = 0 } \\
{ a _ { 3 4 } = 0 }
\end{array} \text { and } \left\{\begin{array}{l}
\left(a_{34}-3 a_{43}\right) b_{33}+b_{43}\left(a_{33}-b_{43}\right)=0 \\
2 a_{43}^{2}+\left(2 a_{33}-a_{34}+b_{43}\right) a_{43}-a_{34}\left(a_{33}-b_{43}\right)=0 \\
a_{34}=0 \\
a_{34}+b_{43}+2 a_{33}=0 .
\end{array}\right.\right.
$$

We get $a_{33}=0, a_{34}=0, a_{43}=0, a_{44}=0, b_{34}=0, b_{43}=0$ and $b_{44}=0$. Then the product in $U^{\star}$ is given by $e_{3} . e_{3}=b_{33} e_{4}$ (who is indeed a left-symmetric product) and the Lie bracket in $U \oplus U^{\star}$ is given by

$$
\left[e_{1}, e_{2}\right]=-e_{1},\left[e_{2}, e_{3}\right]=x e_{1}-e_{3}-e_{4},\left[e_{2}, e_{4}\right]=-e_{4} \text {. }
$$

Proof. of Theorem 1.1.

The Theorem 2.2 confirms that for each Lie algebra $\mathfrak{g}$ of the tables 4 and 5 there exists a base $B_{0}=$ $\left(e_{1}, e_{2}, e_{3}, e_{4}\right)$ such that the para-kähler structure is given by

$$
\omega=e^{13}+e^{24} \text { and } K=E_{11}+E_{22}-E_{33}-E_{44}
$$

and the Lie brackets depend on some parameters. In Tables 6 and 7 we build a family of isomorphisms (depending on the values of parameters) from $\mathfrak{g}\left(\mathfrak{B}_{\mathfrak{i}, j}\right.$ or $\left.\mathcal{C}_{i, j}\right)$ onto a four-dimensional Lie algebra, (say $A$ ) of the Table 1. Each isomorphism is given by the passage matrix $P$ from $B_{0}$ to $B=\left(f_{1}, f_{2}, f_{3}, f_{4}\right)$. The image by $P$ of the para-Kähler structure $(\omega, K)$ is given by the matrices of its component in the bases $B$ and $B^{\star}$ by

$$
{ }^{t} P \circ \omega \circ P=\omega_{i} \text { and } P^{-1} \circ K \circ P=K_{i} .
$$

In this way we collect all the possible para-kähler structures $\left(\omega_{i}, K_{i}\right)$ on $A$. Thereafter, we proceed to the classification in $A$ (up to automorphism).

We will give the proof in the case $\mathfrak{r r}_{3,0}$ since all cases should be handled in a similar way. We will show that the Lie algebra $\mathfrak{r r}_{3,0}$ admits two non-equivalent para-Kähler structures. Note that in this case the non vanishing Lie bracket is

$$
\left[f_{1}, f_{2}\right]=f_{2}
$$

the symplectic form is $\omega_{0}=f^{12}+f^{34}$ and the automorphisms is

$$
T=\left(\begin{array}{cccc}
1 & 0 & 0 & 0 \\
a_{2,1} & a_{2,2} & 0 & 0 \\
a_{3,1} & 0 & a_{3,3} & a_{3,4} \\
a_{4,1} & 0 & a_{4,3} & a_{4,4}
\end{array}\right)
$$

The groups of automorphisms of four-dimensional Lie algebras were given in [10].

From Table 6 and Table 7, $\mathfrak{r r}_{3.0}$ is obtained four times.

1. The transformation: $f_{1}=-e_{4}, f_{2}=e_{2}, f_{3}=e_{3}, f_{4}=e_{1}$ gives an isomorphism from $e_{1,6}$ to $\mathfrak{r r}_{3,0}$ and the para-Kähler structure obtained on $\mathfrak{r r}_{3,0}$ is

$$
\omega_{1}=f^{12}-f^{34} \text { and } K_{1}=-E_{11}+E_{22}-E_{33}+E_{44} .
$$


2. The transformation: $f_{1}=-e_{2}, f_{2}=-y e_{2}+e_{4}, f_{3}=e_{1}, f_{4}=e_{3}$ gives an isomorphism from $e_{2,1}$ with $x=0$ to $\mathfrak{r r}_{3,0}$ and the para-Kähler structure obtained on $\mathfrak{r t}_{3,0}$ is

$$
\omega_{2}=-f^{12}+f^{34} \text { and } K_{2}=E_{11}+2 y E_{12}-E_{22}+E_{33}-E_{44} .
$$

3. The transformation: $f_{1}=-e_{2}, f_{2}=e_{4}, f_{3}=e_{1}, f_{4}=e_{3}$ gives an isomorphism from $\mathcal{C}_{2,2}$ with $x=0, y=0$ to $\mathfrak{r r}_{3,0}$ and the para-Kähler structure obtained on $\mathfrak{r r}_{3,0}$ is

$$
\omega_{3}=-f^{12}+f^{34} \text { and } K_{3}=E_{11}-E_{22}+E_{33}-E_{44} .
$$

4. The transformation: $f_{1}=e_{1}-e_{2}, f_{2}=e_{4}, f_{3}=e_{1}, f_{4}=e_{3}$ gives an isomorphism from $\mathcal{C}_{2,3}$ with $x=0, y=0$ to $\mathfrak{r r}_{3,0}$ and the para-Kähler structure obtained on $\mathfrak{r r}_{3,0}$ is

$$
\omega_{4}=-f^{12}+f^{14}+f^{34} \text { and } K_{4}=E_{11}-E_{22}+E_{33}-E_{44} .
$$

the algebra $\mathfrak{r r}_{3,0}$ supports $\omega_{0}$ as a unique symplectic structure (up to automorphism), therefore there are four families of automorphisms $T_{i}, i \in\{1, \ldots, 4\}$ such that, $T_{i}^{\star} \omega_{i}=\omega_{0}$ for $i \in\{1, \ldots, 4\}$, a direct calculation gives us

$$
\begin{aligned}
T_{1} & =\left(\begin{array}{cccc}
1 & 0 & 0 & 0 \\
a_{2,1} & 1 & 0 & 0 \\
0 & 0 & \frac{a_{3,4} a_{4,3}-1}{a_{4,4}} & a_{3,4} \\
0 & 0 & a_{4,3} & a_{4,4}
\end{array}\right), T_{2}=\left(\begin{array}{cccc}
1 & 0 & 0 & 0 \\
a_{2,1} & -1 & 0 & 0 \\
0 & 0 & \frac{a_{3,4} a_{4,3}+1}{a_{4,4}} & a_{3,4} \\
0 & 0 & a_{4,3} & a_{4,4}
\end{array}\right) \\
T_{3} & =\left(\begin{array}{cccc}
1 & 0 & 0 & 0 \\
a_{2,1} & 1 & 0 & 0 \\
0 & 0 & \frac{a_{3,4} a_{4,3}-1}{a_{4,4}} & a_{3,4} \\
0 & 0 & a_{4,3} & a_{4,4}
\end{array}\right), T_{4}=\left(\begin{array}{cccc}
1 & 0 & 0 & 0 \\
a_{2,1} & -1 & 0 & 0 \\
-1 & 0 & \frac{a_{3,4} a_{4,3}+1}{a_{4,4}} & a_{3,4} \\
0 & 0 & a_{4,3} & a_{4,4}
\end{array}\right) .
\end{aligned}
$$

Thus we obtain four para-Kähler structures on $\mathfrak{r r}_{3,0}$ given by $\left(\omega_{0}, K_{0 i}\right), i \in 1, \ldots, 4$ with $K_{0 i}=T_{i}^{-1} \circ K_{i} \circ T_{i}$ a direct calculation gives us

$$
\begin{aligned}
& K_{01}=-E_{11}+E_{22}-E_{33}+E_{44} \\
& K_{02}=E_{11}+2 y E_{12}-E_{22}+E_{33}-E_{44} \\
& K_{03}=E_{11}-E_{22}+E_{33}-E_{44} \\
& K_{04}=-E_{11}+E_{22}+E_{33}-E_{44}
\end{aligned}
$$

We note that $K_{03}$ is a sub-case of $K_{02}$ and $\left(\omega_{0}, K_{04}\right)$ is isomorphic to $\left(\omega_{0}, K_{01}\right)$. Indeed, we have $L^{\star} \omega_{0}=\omega_{0}$ and $L^{-1} \circ K_{04} \circ L=K_{01}$ with

$$
L=\left(\begin{array}{cccc}
1 & 0 & 0 & 0 \\
0 & 1 & 0 & 0 \\
0 & 0 & 0 & 1 \\
0 & 0 & -1 & 0
\end{array}\right)
$$

We complete the proof by showing that $\left(\omega_{0}, K_{01}\right)$ is not isomorphic to $\left(\omega_{0}, K_{02}\right)$. In fact, the symplectomorphism group of $\omega_{0}$ is generated by

$$
L_{1}=\left(\begin{array}{cccc}
1 & 0 & 0 & 0 \\
a_{2,1} & 1 & 0 & 0 \\
0 & 0 & \frac{a_{3,4} a_{4,3}+1}{a_{4,4}} & a_{3,4} \\
0 & 0 & a_{4,3} & a_{4,4}
\end{array}\right) \quad \text { and } \quad L_{2}=\left(\begin{array}{cccc}
1 & 0 & 0 & 0 \\
a_{2,1} & 1 & 0 & 0 \\
0 & 0 & a_{3,3} & a_{3,4} \\
0 & 0 & -a_{3,4}{ }^{-1} & 0
\end{array}\right) \text {. }
$$

A simple calculation gives us

$$
f^{2}\left(\left(L_{1}^{-1} \circ K_{01} \circ L_{1}-K_{02}\right)\left(f_{2}\right)\right)=2 \quad \text { and } \quad f^{1}\left(\left(L_{2}^{-1} \circ K_{01} \circ L_{2}-K_{02}\right)\left(f_{1}\right)\right)=-2
$$

so

$$
L_{1}^{-1} \circ K_{01} \circ L_{1}=K_{02} \quad \text { and } \quad L_{2}^{-1} \circ K_{01} \circ L_{2} /=K_{02} .
$$




\section{Application: Curvatures properties of four-dimensional para-Kähler Lie algebras}

Now let $(\mathfrak{g}, \omega, K)$ denote a four-dimensional para-Kähler Lie algebra. Let $\nabla: \mathfrak{g} \times \mathfrak{g} \longrightarrow \mathfrak{g}$ be the Levi-Civita product associated to a left-invariant pseudo-Riemannian metric $h(X, Y)=\omega(K X, Y)$. The connection $\nabla$ is also called Hess connection. The curvature tensor is then described in terms of the map

$$
\begin{aligned}
R: \mathfrak{g} \times \mathfrak{g} & \longrightarrow g l(\mathfrak{g}) \\
(X, Y) & \longmapsto R(X, Y)=\nabla_{[X, Y]}-\left[\nabla_{X}, \nabla_{Y}\right]
\end{aligned} .
$$

The Ricci tensor is the symmetric tensor ric given by $\operatorname{ric}(X, Y)=\operatorname{tr}(Z \longmapsto R(X, Z) Y)$ and the Ricci operator Ric $: \mathfrak{g} \longrightarrow \mathfrak{g}$ is given by the relation $h(\operatorname{Ric}(X), Y)=\operatorname{ric}(X, Y)$. The scalar curvature is defined in the standard way by $s=\operatorname{tr}($ Ric $)$.

Recall that: $(\mathfrak{g}, h)$ is called flat if $R=0$, Ricci flat if Ric $=0$ and Ricci soliton if

$$
\mathcal{L}_{X} h+r i c=\lambda h,
$$

where $X=x_{1} e_{1}+x_{2} e_{2}+x_{3} e_{3}+x_{4} e_{4}$ is a vector field and $\lambda$ is a real constant, in that case if $X=0$ then $h$ is called Einstein metric and if $\lambda$ is positive, zero, or negative then $h$ is called a shrinking, steady, or expanding Ricci soliton, respectively. We give in the following theorem some geometrical situations for the left invariant four-dimensional dimensional para-Kähler Lie groups.

Remark 3. Opposite metrics have the same Levi-Civita product and the same curvature and Ricci tensors. Thus, for a metric $h$ and its opposite $g=-h$, one has that

$$
\mathcal{L}_{X} g+r i c^{g}=\lambda g \Leftrightarrow \mathcal{L}_{X}(-h)+r i c^{h}=\lambda(-h) \Leftrightarrow \mathcal{L}_{-X} h+r i c^{h}=-\lambda h,
$$

Theorem 3.1. Let $(\mathfrak{g}, \omega, K)$ be a class of para-Kähler Lie algebras obtained in Theorem 1.1. The associated para-Kähler metric (up to sign) and some of its properties are given in the following table

Table 3: Curvature properties of four-dimensional para-Kähler Lie algebras

\begin{tabular}{clcccc}
\hline \multirow{2}{*}{ Lie algebra } & \multirow{2}{*}{ Para-Kähler metric } & \multirow{2}{*}{$R=0$} & Ric $=0$ & \multicolumn{2}{c}{ Ricci soliton } \\
& & & & $\lambda$ \\
\hline \multirow{2}{*}{$\mathfrak{r h}_{3}$} & $\dot{e}^{14}-\dot{e}^{23}$ & Yes & Yes & 0 & $\left(0,0, x_{3}, x_{4}\right)$ \\
& $\dot{e}^{13}-\dot{e}^{14}+\dot{e}^{23}$ & Yes & Yes & 0 & $\left(0,0, x_{3}, x_{4}\right)$ \\
\hline \multirow{2}{*}{$\mathfrak{r r}_{3,0}$} & $-\dot{e}^{12}-\dot{e}^{34}$ & Yes & Yes & 0 & $\left(0,0, x_{3}, x_{4}\right)$ \\
& $\dot{e}^{12}+x \dot{e}^{22}+\dot{e}^{34}, x \neq 0$ & No & No & & No \\
\hline \multirow{2}{*}{$\mathfrak{r v}_{3,-1}$} & $-\dot{e}^{14}+\dot{e}^{23}$ & Yes & Yes & 0 & $\left(x_{1}, 0,0, x_{4}\right)$ \\
& $\dot{e}^{14}+\dot{e}^{23} \pm \dot{e}^{33}$ & No & Yes & 0 & $\left(0,0,0, x_{4}\right)$ \\
& $\dot{e}^{14}+\dot{e}^{23}+x \dot{e}^{44}, x \neq 0$ & Yes & Yes & 0 & $\left(x_{1}, 0,0, x_{4}\right)$
\end{tabular}




\begin{tabular}{|c|c|c|c|c|c|}
\hline & $-\dot{e}^{14} \pm \dot{e}^{22}+\dot{e}^{23}$ & No & Yes & 0 & $\left(0,0,0, x_{4}\right)$ \\
\hline \multirow{6}{*}{$\begin{array}{c}\mathfrak{r}_{2} \mathfrak{r}_{2} \\
\mu>0\end{array}$} & $-\dot{e}^{12}-\mu \dot{e}^{13}+\dot{e}^{34}$ & No & Yes & 0 & $(0,0,0,0)$ \\
\hline & $\dot{e}^{12}+\mu \dot{e}^{13}-2 \dot{e}^{23}-2 \mu \dot{e}^{33}-\dot{e}^{34}$ & No & Yes & 0 & $(0,0,0,0)$ \\
\hline & $\dot{e}^{12}+2 \dot{e}^{14}-\dot{e}^{34}$ & Yes & Yes & $-x_{3}$ & $\left(x_{3}, 0, x_{3}, 0\right)$ \\
\hline & $-\dot{e}^{12}+x \dot{e}^{22}+\dot{e}^{23}+x \dot{e}^{24}-\frac{1}{x} \dot{e}^{33}+x \dot{e}^{44}, x \neq 0$ & No & No & $\frac{3}{2} x$ & $(0,0,0,0)$ \\
\hline & $\dot{e}^{12}+x \dot{e}^{22}+\dot{e}^{34}+y \dot{e}^{44}, x y \neq 0, x \neq y$ & No & No & & No \\
\hline & $\dot{e}^{12}+x \dot{e}^{22}+\dot{e}^{34}+x \dot{e}^{44}, x \neq 0$ & No & No & $x$ & $(0,0,0,0)$ \\
\hline \multirow[t]{2}{*}{$\mathfrak{r}_{2} \mathfrak{r}_{2}$} & $-\dot{e}^{12}+\dot{e}^{34}+x \dot{e}^{44}, x \neq 0$ & No & No & $x$ & $(-x, 0,0,0)$ \\
\hline & $-\dot{e}^{12}+\dot{e}^{34}$ & Yes & Yes & $-x_{3}$ & $\left(x_{3}, 0, x_{3}, 0\right)$ \\
\hline \multirow[t]{5}{*}{$\mu=0$} & $\dot{e}^{12}-2 \dot{e}^{23}-\dot{e}^{34}$ & Yes & Yes & $-x_{3}$ & $\left(x_{3}, 0, x_{3}, 0\right)$ \\
\hline & $\dot{e}^{12}+x \dot{e}^{22}+x \dot{e}^{24}+\dot{e}^{34}+x \dot{e}^{44}, x /=0$ & No & No & $\frac{3}{2} x$ & $(0,0,0,0)$ \\
\hline & $\dot{e}^{12}+\dot{e}^{34}$ & Yes & Yes & $-x_{3}$ & $\left(x_{3}, 0, x_{3}, 0\right)$ \\
\hline & $\dot{e}^{14}+\frac{4}{x} \dot{e}^{22}-\dot{e}^{23}+x \dot{e}^{44}, x \neq 0$ & No & No & $\frac{3}{2} x$ & $(0,0,0,0)$ \\
\hline & $h_{1}, \quad x y /=0$ & No & No & & No \\
\hline \multirow[t]{6}{*}{$\mathfrak{r}_{2}^{\prime}$} & $h_{2}, \quad y /=0$ & No & No & & No \\
\hline & $h_{3}, \quad y /=0$ & No & No & $-4 y$ & $(0,0,0,0)$ \\
\hline & $-(1+x) \dot{e}^{12}+x\left(\dot{e}^{14}+\dot{e}^{23}\right)+(1-x) \dot{e}^{34}, x /=0,1$ & No & No & & No \\
\hline & $-2 \dot{e}^{12}+\dot{e}^{14}+\dot{e}^{23}$ & Yes & Yes & $-x_{3}$ & $\left(x_{3}, 0, x_{3}, 0\right)$ \\
\hline & $-\dot{e}^{12}+\dot{e}^{34}$ & No & No & & No \\
\hline & $-\dot{e}^{14}-\dot{e}^{23}$ & Yes & Yes & $-x_{1}$ & $\left(x_{1}, 0,0,0\right)$ \\
\hline $\mathfrak{r}_{4,0}$ & $\dot{e}^{14}-\dot{e}^{23}$ & No & Yes & 0 & $\left(0, x_{2}, 0,0\right)$ \\
\hline \multirow[b]{2}{*}{$\mathfrak{r}_{4,-1}$} & $\dot{e}^{13}-\dot{e}^{24}+x \dot{e}^{33}, x /=0$ & No & Yes & 0 & $(0,0,0,0)$ \\
\hline & $\dot{e}^{13}-\dot{e}^{24}$ & Yes & Yes & 0 & $(0,0,0,0)$ \\
\hline \multirow{6}{*}{$\mathfrak{r}_{4,-1, \beta}$} & $\dot{e}^{12}+\dot{e}^{22}-\dot{e}^{34}, \beta /=0$ & No & Yes & 0 & $(0,0,0,0)$ \\
\hline & $\dot{e}^{12}+\dot{e}^{22}-\dot{e}^{34}, \beta=0$ & No & Yes & 0 & $\left(0,0, x_{3}, 0\right)$ \\
\hline & $\dot{e}^{12} \pm \dot{e}^{34}, \beta /=0$ & Yes & Yes & 0 & $(0,0,0,0)$ \\
\hline & $\dot{e}^{12} \pm \dot{e}^{34}, \beta=0$ & Yes & Yes & 0 & $\left(0,0, x_{3}, x_{4}\right)$ \\
\hline & $\dot{e}^{12}-\frac{1}{x} \dot{e}^{33}+x \dot{e}^{44}, \beta /=0$ & No & No & & No \\
\hline & $\dot{e}^{12}-\frac{1}{x} \dot{e}^{33}+x \dot{e}^{44}, \beta=0$ & Yes & Yes & 0 & $\left(0,0, x_{3}, x_{4}\right)$ \\
\hline \multirow{6}{*}{$\mathfrak{r}_{4,-1,-1}$} & $\pm \dot{e}^{11}-\dot{e}^{12}-\dot{e}^{34}$ & No & Yes & 0 & $(0,0,0,0)$ \\
\hline & $\pm \dot{e}^{12}-x \dot{e}^{33}-\dot{e}^{34}, x /=0$ & No & No & & No \\
\hline & $\pm \dot{e}^{12}-\dot{e}^{34}$ & Yes & Yes & 0 & $(0,0,0,0)$ \\
\hline & $\dot{e}^{12} \pm \dot{e}^{22}-\dot{e}^{34}$ & No & Yes & 0 & $(0,0,0,0)$ \\
\hline & $-\chi \dot{e}^{11}-\dot{e}^{12}-\dot{e}^{13}-\dot{e}^{34}, \chi /=0$ & No & Yes & 0 & $(0,0,0,0)$ \\
\hline & $-\dot{e}^{12}-\dot{e}^{13}-\dot{e}^{34}$ & Yes & Yes & 0 & $(0,0,0,0)$ \\
\hline \multirow{4}{*}{$\mathfrak{r}_{4, \alpha,-\alpha}$} & $-\dot{e}^{14}+\dot{e}^{23} \pm \dot{e}^{33}$ & No & Yes & 0 & $(0,0,0,0)$ \\
\hline & $-\chi \dot{e}^{11}-\dot{e}^{14}+\dot{e}^{23}, x /=0$ & No & No & & No \\
\hline & $\pm \dot{e}^{14}+\dot{e}^{23}$ & Yes & Yes & 0 & $(0,0,0,0)$ \\
\hline & $\dot{e}^{14} \pm \dot{e}^{22}+\dot{e}^{23}$ & No & Yes & 0 & $(0,0,0,0)$ \\
\hline \multirow{11}{*}{$\mathfrak{d}_{4,1}$} & $\dot{e}^{12} \pm \dot{e}^{22}+\dot{e}^{34}$ & Yes & Yes & 0 & $\left(x_{1}, 0,0,0\right)$ \\
\hline & $\dot{e}^{12}+x \dot{e}^{33}+\dot{e}^{34}, x /=0$ & No & No & $\frac{3}{2} x$ & $(0,0,0,0)$ \\
\hline & $\dot{e}^{12}+\dot{e}^{34}$ & Yes & Yes & $-x_{4}$ & $\left(x_{1}, 0,0, x_{4}\right)$ \\
\hline & $-\dot{e}^{12}+x \dot{e}^{33}+\dot{e}^{34}, x /=0$ & No & No & & No \\
\hline & $-\dot{e}^{12}+\dot{e}^{34}$ & Yes & Yes & 0 & $(0,0,0,0)$ \\
\hline & $-\dot{e}^{11}+\dot{e}^{12}-x \dot{e}^{13}-\dot{e}^{34}, x /=0$ & No & No & & No \\
\hline & $-\dot{e}^{11}+\dot{e}^{12}-\dot{e}^{34}$ & No & Yes & 0 & $(0,0,0,0)$ \\
\hline & $\pm \dot{e}^{11}-\dot{e}^{12}+\dot{e}^{34}$ & No & Yes & 0 & $(0,0,0,0)$ \\
\hline & $\dot{e}^{12}+x \dot{e}^{22}-\dot{e}^{24}+\dot{e}^{34}$ & Yes & Yes & 0 & $\left(x_{1}, 0,0,0\right)$ \\
\hline & $\dot{e}^{12}+x \dot{e}^{22}+\dot{e}^{34}, x \neq 0$ & No & Yes & 0 & $\left(x x_{2}, x_{2}, 0,0\right)$ \\
\hline & $\dot{e}^{12}+\dot{e}^{34}$ & Yes & Yes & $-x_{4}$ & $\left(0, x_{2}, 0, x_{4}\right)$ \\
\hline
\end{tabular}




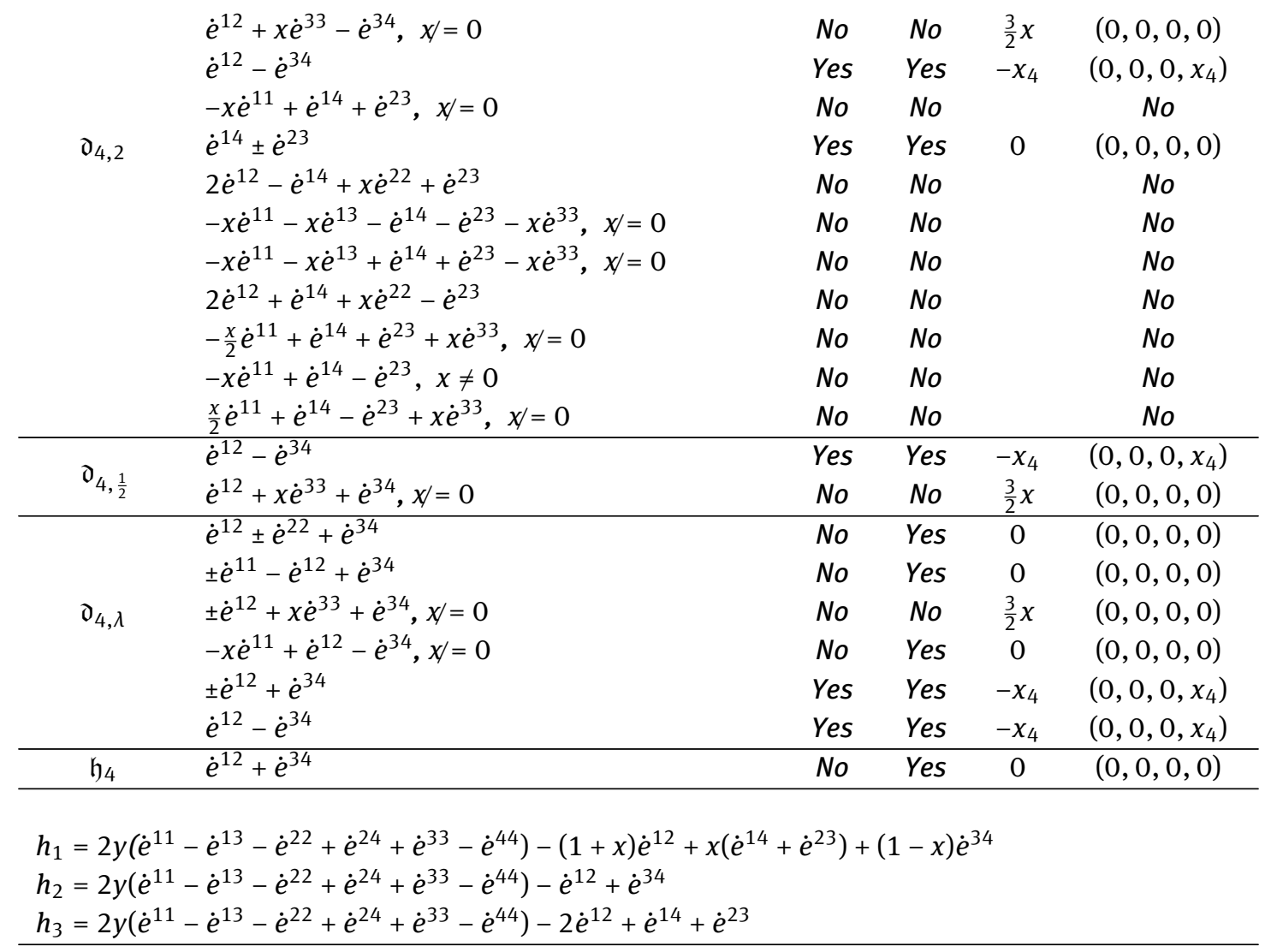

Proof. We report below the details for the case of $\mathfrak{d}_{4, \frac{1}{2}}$ the other cases are treated in the same way. Let $\left\{e_{1}, e_{2}, e_{3}, e_{4}\right\}$ denote the basis used in theorem 1.1 for $\mathfrak{d}_{4, \frac{1}{2}}$. The non isomorphic para-Kähler structures in $\mathfrak{d}_{4, \frac{1}{2}}$ are $\left(\omega, K_{1}\right)$ and $\left(\omega, K_{2}\right)$ where $\omega=e^{12}-e^{34}: K_{1}=E_{11}-E_{22}-E_{33}+x E_{43}+E_{44}, \quad x \neq 0$ and $K_{2}=E_{11}-E_{22}+E_{33}-E_{44}$.

The corresponding compatible metric to $\left(\omega, K_{i}\right)$ is uniquely determined by $h_{i}(X, Y)=\omega\left(K_{i} X, Y\right)$. Hence, para-Kähler metrics in $\mathfrak{d}_{4, \frac{1}{2}}$ are of the form :

$$
h_{1}=\left(\begin{array}{cccc}
0 & 1 & 0 & 0 \\
1 & 0 & 0 & 0 \\
0 & 0 & x & 1 \\
0 & 0 & 1 & 0
\end{array}\right) \quad x \in \mathbb{R}^{*}, \quad h_{2}=\left(\begin{array}{cccc}
0 & 1 & 0 & 0 \\
1 & 0 & 0 & 0 \\
0 & 0 & 0 & -1 \\
0 & 0 & -1 & 0
\end{array}\right) .
$$

- For $h_{1}$, using the Koszul formula the Levi-Civita connection is described by

$$
\begin{array}{ll}
\nabla_{e_{1}}=\left(\begin{array}{cccc}
0 & 0 & -\frac{1}{2} x & -1 \\
0 & 0 & 0 & 0 \\
0 & 1 & 0 & 0 \\
0 & -\frac{1}{2} x & 0 & 0
\end{array}\right), & \nabla_{e_{2}}=\left(\begin{array}{cccc}
0 & 0 & 0 & 0 \\
0 & 0 & \frac{1}{2} x & 0 \\
0 & 0 & 0 & 0 \\
-\frac{1}{2} x & 0 & 0 & 0
\end{array}\right) . \\
\nabla_{e_{3}}=\left(\begin{array}{cccc}
-\frac{1}{2} x & 0 & 0 & 0 \\
0 & \frac{1}{2} x & 0 & 0 \\
0 & 0 & x & 0 \\
0 & 0 & -x^{2} & -x
\end{array}\right), & \nabla_{e_{4}}=\left(\begin{array}{cccc}
-\frac{1}{2} & 0 & 0 & 0 \\
0 & \frac{1}{2} & 0 & 0 \\
0 & 0 & 1 & 0 \\
0 & 0 & -x & -1
\end{array}\right) .
\end{array}
$$


Then, we calculate the curvature matrices $R\left(e_{i}, e_{j}\right)$ (for $\left.1 \leq i<j \leq 4\right)$ and we find

$$
\begin{gathered}
R\left(e_{1}, e_{2}\right)=\left(\begin{array}{cccc}
-x & 0 & 0 & 0 \\
0 & x & 0 & 0 \\
0 & 0 & \frac{x}{2} & 0 \\
0 & 0 & -\frac{1}{2} x^{2} & -\frac{1}{2} x
\end{array}\right), \quad R\left(e_{1}, e_{3}\right)=\left(\begin{array}{cccc}
0 & 0 & -\frac{1}{4} x^{2} & -\frac{x}{2} \\
0 & 0 & 0 & 0 \\
0 & \frac{x}{2} & 0 & 0 \\
0 & -\frac{1}{4} x^{2} & 0 & 0
\end{array}\right) \\
R\left(e_{2}, e_{3}\right)=\left(\begin{array}{cccc}
0 & 0 & 0 & 0 \\
0 & 0 & -\frac{1}{4} x^{2} & 0 \\
0 & 0 & 0 & 0 \\
\frac{1}{4} x^{2} & 0 & 0 & 0
\end{array}\right), \quad R\left(e_{2}, e_{4}\right)=\left(\begin{array}{cccc}
0 & 0 & 0 & 0 \\
0 & 0 & -\frac{x}{2} & 0 \\
0 & 0 & 0 & 0 \\
\frac{x}{2} & 0 & 0 & 0
\end{array}\right) \\
R\left(e_{3}, e_{4}\right)=\left(\begin{array}{cccc}
\frac{x}{2} & 0 & 0 & 0 \\
0 & -\frac{x}{2} & 0 & 0 \\
0 & 0 & -x & 0 \\
0 & 0 & x^{2} & x
\end{array}\right) \text { and } R\left(e_{1}, e_{4}\right)=0 .
\end{gathered}
$$

The Ricci tensor ric and the Ricci operator Ric are given by

$$
\text { ric }=\left(\begin{array}{cccc}
0 & \frac{3}{2} x & 0 & 0 \\
\frac{3}{2} x & 0 & 0 & 0 \\
0 & 0 & \frac{3}{2} x^{2} & \frac{3}{2} x \\
0 & 0 & \frac{3}{2} x & 0
\end{array}\right) \quad \text { and } \quad \text { Ric }=\left(\begin{array}{cccc}
\frac{3}{2} x & 0 & 0 & 0 \\
0 & \frac{3}{2} x & 0 & 0 \\
0 & 0 & \frac{3}{2} x & 0 \\
0 & 0 & 0 & \frac{3}{2} x
\end{array}\right) \text {. }
$$

The Lie derivative $\mathcal{L}_{X} h_{1}$ of the metric $h_{1}$ with respect to an arbitrary vector field $X=x_{1} e_{1}+x_{2} e_{2}+x_{3} e_{3}+x_{4} e_{4} \in$ $\mathfrak{g}$ is given by

$$
\mathcal{L}_{X} h_{1}=\left(\begin{array}{cccc}
0 & -x_{4} & x_{2} x & \frac{3}{2} x_{2} \\
-x_{4} & 0 & -x_{1} x & -\frac{1}{2} x_{1} \\
x_{2} x & -x_{1} x & -2 x_{4} x & x x_{3}-x_{4} \\
\frac{3}{2} x_{2} & -\frac{1}{2} x_{1} & x x_{3}-x_{4} & 2 x_{3}
\end{array}\right) .
$$

Then, solving equation $\mathcal{L}_{X} h_{1}+$ ric $=\lambda h_{1}, x /=0$ we obtain

$$
\lambda=\frac{3}{2} x \quad \text { and } \quad X=0 .
$$

Notice that, in this case the para-Kähler metric is an Einstein metric not Ricci flat.

- For $h_{2}$

$$
\nabla e_{2} e_{1}=-e_{3}, \nabla e_{2} e_{4}=-e_{2}, \nabla e_{4} e_{1}=\frac{1}{2} e_{1}, \nabla e_{4} e_{2}=-\frac{1}{2} e_{2}, \nabla e_{4} e_{3}=e_{3}, \nabla e_{4} e_{4}=-e_{4} .
$$

This para-Kähler structure is flat $\left(R\left(e_{i}, e_{j}\right)=0\right.$ for $\left.1 \leq i<j \leq 4\right)$ and also Ricci flat. The Lie derivative $\mathcal{L}_{X} h_{2}$ of the metric $h_{2}$ is given by

$$
\mathcal{L}_{X} h_{2}=\left(\begin{array}{cccc}
0 & -x_{4} & 0 & -\frac{1}{2} x_{2} \\
-x_{4} & 0 & 0 & \frac{3}{2} x_{1} \\
0 & 0 & 0 & x_{4} \\
-\frac{1}{2} x_{2} & \frac{3}{2} x_{1} & x_{4} & -2 x_{3}
\end{array}\right) .
$$

Then, solving equation $\mathcal{L}_{X} h_{2}+$ ric $=\lambda h_{2}$, we obtain

$$
\lambda=-x_{4} \quad \text { and } \quad X=x_{4} e_{4} .
$$




\section{Tables}

Table 4: Four-dimensional Para-Kähler Lie algebras coming from $\mathfrak{b}$

\begin{tabular}{|c|c|}
\hline Lie algebra & No zero brackets \\
\hline $\mathcal{B}_{1, \alpha}^{1} \alpha / \in\{-2,-1,1\}$ & {$\left[e_{1}, e_{2}\right]=-e_{1},\left[e_{2}, e_{3}\right]=x e_{1}-e_{3},\left[e_{2}, e_{4}\right]=-\alpha e_{4}$} \\
\hline $\mathcal{B}_{1, \alpha}^{2} \alpha / \in\{-2,-1,0,1\}$ & {$\left[e_{1}, e_{2}\right]=-e_{1},\left[e_{1}, e_{4}\right]=-\frac{\chi}{\alpha} e_{1},\left[e_{2}, e_{3}\right]=-e_{3},\left[e_{2}, e_{4}\right]=x e_{2}-\alpha e_{4},\left[e_{3}, e_{4}\right]=\frac{\chi}{\alpha} e_{3}$} \\
\hline $\mathcal{B}_{1,-2}^{1}$ & {$\left[e_{1}, e_{2}\right]=-e_{1},\left[e_{1}, e_{4}\right]=x e_{1},\left[e_{2}, e_{3}\right]=y e_{1}-e_{3},\left[e_{2}, e_{4}\right]=2 x e_{2}+2 e_{4},\left[e_{3}, e_{4}\right]=-x e_{3}$} \\
\hline $\mathcal{B}_{1,-2}^{2}$ & {$\left[e_{1}, e_{2}\right]=-e_{1},\left[e_{1}, e_{3}\right]=x e_{1},\left[e_{2}, e_{3}\right]=y e_{1}-x e_{2}-e_{3},\left[e_{2}, e_{4}\right]=x e_{1}+2 e_{4},\left[e_{3}, e_{4}\right]=-2 x e_{4}$} \\
\hline $\mathcal{B}_{1,-1}^{1}$ & {$\left[e_{1}, e_{2}\right]=-e_{1},\left[e_{1}, e_{3}\right]=-x e_{1},\left[e_{2}, e_{3}\right]=y e_{1}+x e_{2}-e_{3},\left[e_{2}, e_{4}\right]=e_{4},\left[e_{3}, e_{4}\right]=x e_{4}$} \\
\hline $\mathcal{B}_{1,-1}^{2}$ & {$\left[e_{1}, e_{2}\right]=-e_{1},\left[e_{1}, e_{4}\right]=x e_{1},\left[e_{2}, e_{3}\right]=-e_{3},\left[e_{2}, e_{4}\right]=x e_{2}+e_{4},\left[e_{3}, e_{4}\right]=-x e_{3}$} \\
\hline $\mathcal{B}_{1,0}$ & {$\left[e_{1}, e_{2}\right]=-e_{1},\left[e_{1}, e_{4}\right]=x e_{1},\left[e_{2}, e_{3}\right]=-e_{3},\left[e_{3}, e_{4}\right]=-x e_{3}$} \\
\hline $\mathcal{B}_{1,1}^{1} \quad x /=0$ & $\begin{array}{l}{\left[e_{1}, e_{2}\right]=-e_{1},\left[e_{1}, e_{3}\right]=-\frac{y}{2} e_{1},\left[e_{1}, e_{4}\right]=-x e_{1},\left[e_{2}, e_{3}\right]=\frac{y^{2}}{2 x} e_{1}+\frac{y}{2} e_{2}-e_{3}} \\
{\left[e_{2}, e_{4}\right]=y e_{1}+x e_{2}-e_{4},\left[e_{3}, e_{4}\right]=x e_{3}-\frac{y}{2} e_{4}}\end{array}$ \\
\hline$\overline{\mathcal{B}_{1,1}^{2}}$ & {$\left[e_{1}, e_{2}\right]=-e_{1},\left[e_{2}, e_{3}\right]=x e_{1}-e_{3},\left[e_{2}, e_{4}\right]=-e_{4}$} \\
\hline $\mathcal{B}_{2}$ & {$\left[e_{1}, e_{2}\right]=-e_{1},\left[e_{2}, e_{3}\right]=x e_{1}-e_{3}-e_{4},\left[e_{2}, e_{4}\right]=-e_{4}$} \\
\hline $\mathcal{B}_{3, \alpha}^{1} \alpha \neq 0$ & {$\left[e_{1}, e_{2}\right]=\frac{1}{\alpha} e_{1},\left[e_{1}, e_{3}\right]=\left[e_{2}, e_{4}\right]=-e_{4},\left[e_{2}, e_{3}\right]=x e_{1}+\frac{1-\alpha}{\alpha} e_{3}$} \\
\hline $\mathcal{B}_{3, \alpha}^{2} \alpha \neq 0$ & {$\left[e_{1}, e_{2}\right]=\frac{1}{\alpha} e_{1},\left[e_{1}, e_{3}\right]=\left[e_{2}, e_{4}\right]=x \alpha e_{2}-e_{4},\left[e_{1}, e_{4}\right]=x e_{1},\left[e_{2}, e_{3}\right]=\frac{1-\alpha}{\alpha} e_{3},\left[e_{3}, e_{4}\right]=x(\alpha-1) e_{3}$} \\
\hline$\overline{\mathcal{B}_{3, \frac{1}{2}}^{1}}$ & {$\left[e_{1}, e_{2}\right]=2 e_{1},\left[e_{1}, e_{3}\right]=x e_{1}-e_{4},\left[e_{2}, e_{3}\right]=y e_{1}-\frac{x}{2} e_{2}+e_{3},\left[e_{2}, e_{4}\right]=-e_{4},\left[e_{3}, e_{4}\right]=-\frac{x}{2} e_{4}$} \\
\hline $\mathcal{B}_{3, \frac{1}{2}}^{2} y \neq 0$ & $\begin{array}{l}{\left[e_{1}, e_{2}\right]=2 e_{1},\left[e_{1}, e_{3}\right]=-2 x e_{1}+\frac{y}{2} e_{2}-e_{4},\left[e_{1}, e_{4}\right]=y e_{1},\left[e_{2}, e_{3}\right]=-\frac{3 x^{2}}{y} e_{1}+\frac{x}{2} e_{2}+e_{3}} \\
{\left[e_{2}, e_{4}\right]=x e_{1}+\frac{y}{2} e_{2}-e_{4},\left[e_{3}, e_{4}\right]=-\frac{y}{2} e_{3}-\frac{x}{2} e_{4}}\end{array}$ \\
\hline$\overline{\mathcal{B}_{3, \frac{1}{2}}^{3}}$ & {$\left[e_{1}, e_{2}\right]=2 e_{1},\left[e_{1}, e_{3}\right]=\left[e_{2}, e_{4}\right]=x e_{1}-e_{4},\left[e_{2}, e_{3}\right]=y e_{1}-x e_{2}+e_{3},\left[e_{3}, e_{4}\right]=-2 x e_{4}$} \\
\hline $\mathcal{B}_{3,1}$ & {$\left[e_{1}, e_{2}\right]=e_{1},\left[e_{1}, e_{3}\right]=x e_{1}+y e_{2}-e_{4},\left[e_{1}, e_{4}\right]=y e_{1},\left[e_{2}, e_{3}\right]=z e_{1},\left[e_{2}, e_{4}\right]=y e_{2}-e_{4}$} \\
\hline $\mathcal{B}_{4}$ & {$\left[e_{1}, e_{2}\right]=e_{1},\left[e_{1}, e_{3}\right]=x e_{1}-e_{4},\left[e_{2}, e_{3}\right]=y e_{1}-e_{4},\left[e_{2}, e_{4}\right]=-e_{4}$} \\
\hline$\overline{\mathcal{B}_{5,1}^{+}}$ & {$\left[e_{1}, e_{2}\right]=e_{1},\left[e_{1}, e_{4}\right]=x e_{1}-e_{3},\left[e_{2}, e_{3}\right]=e_{3},\left[e_{2}, e_{4}\right]=-2 x e_{2}+2 e_{4},\left[e_{3}, e_{4}\right]=-x e_{3}$} \\
\hline $\mathcal{B}_{5,2}^{+}$ & {$\left[e_{1}, e_{2}\right]=e_{1},\left[e_{1}, e_{4}\right]=-\frac{x}{4} e_{1}-e_{3},\left[e_{2}, e_{3}\right]=x e_{1}+e_{3},\left[e_{2}, e_{4}\right]=-\frac{x}{2} e_{2}+2 e_{4},\left[e_{3}, e_{4}\right]=\frac{x}{4} e_{3}$} \\
\hline $\mathcal{B}_{5,3}^{+}$ & $\begin{array}{l}{\left[e_{1}, e_{2}\right]=e_{1},\left[e_{1}, e_{3}\right]=-x e_{1},\left[e_{1}, e_{4}\right]=-2 x e_{1}-x e_{2}-e_{3},\left[e_{2}, e_{3}\right]=2 x e_{1}+x e_{2}+e_{3}} \\
{\left[e_{2}, e_{4}\right]=3 x e_{1}+2 x e_{2}+2 e_{4},\left[e_{3}, e_{4}\right]=2 x e_{3}-2 x e_{4}}\end{array}$ \\
\hline$\overline{\mathcal{B}_{5,4}^{+}}$ & $\begin{array}{l}{\left[e_{1}, e_{2}\right]=e_{1},\left[e_{1}, e_{3}\right]=-x e_{1},\left[e_{1}, e_{4}\right]=2 x e_{1}-x e_{2}-e_{3}} \\
{\left[e_{2}, e_{3}\right]=-2 x e_{1}+x e_{2}+e_{3},\left[e_{2}, e_{4}\right]=3 x e_{1}-2 x e_{2}+2 e_{4},\left[e_{3}, e_{4}\right]=-2 x e_{3}-2 x e_{4}}\end{array}$ \\
\hline $\mathcal{B}_{5,1}^{-}$ & {$\left[e_{1}, e_{2}\right]=e_{1},\left[e_{1}, e_{4}\right]=x e_{1}+e_{3},\left[e_{2}, e_{3}\right]=e_{3},\left[e_{2}, e_{4}\right]=-2 x e_{2}+2 e_{4},\left[e_{3}, e_{4}\right]=-x e_{3}$} \\
\hline $\mathcal{B}_{5,2}^{-}$ & {$\left[e_{1}, e_{2}\right]=e_{1},\left[e_{1}, e_{4}\right]=\frac{x}{4} e_{1}+e_{3},\left[e_{2}, e_{3}\right]=x e_{1}+e_{3},\left[e_{2}, e_{4}\right]=\frac{x}{2} e_{2}+2 e_{4},\left[e_{3}, e_{4}\right]=-\frac{x}{4} e_{3}$} \\
\hline
\end{tabular}


Table 5: Four-dimensional Para-Kähler Lie algebras coming from $c$

\begin{tabular}{|c|c|}
\hline Lie algebra & No zero brackets \\
\hline $\mathrm{e}_{1,1}$ & {$\left[e_{1}, e_{4}\right]=e_{1},\left[e_{2}, e_{4}\right]=\alpha e_{2},\left[e_{3}, e_{4}\right]=-e_{3}$} \\
\hline $\mathrm{e}_{1,2}$ & {$\left[e_{1}, e_{4}\right]=e_{1}+e_{2},\left[e_{2}, e_{4}\right]=e_{2},\left[e_{3}, e_{4}\right]=-e_{3}$} \\
\hline $\mathrm{e}_{1,3}$ & {$\left[e_{1}, e_{3}\right]=\left[e_{2}, e_{4}\right]=e_{2},\left[e_{1}, e_{4}\right]=\left(1-\frac{1}{\alpha}\right) e_{1},\left[e_{3}, e_{4}\right]=\frac{1}{\alpha} e_{3}$} \\
\hline $\mathrm{e}_{1,4}$ & {$\left[e_{1}, e_{3}\right]=\left[e_{1}, e_{4}\right]=\left[e_{2}, e_{4}\right]=e_{2},\left[e_{3}, e_{4}\right]=e_{3}$} \\
\hline$\overline{\mathrm{e}_{1,5}^{+}}$ & {$\left[e_{1}, e_{4}\right]=-e_{1},\left[e_{2}, e_{3}\right]=e_{1},\left[e_{2}, e_{4}\right]=-2 e_{2},\left[e_{3}, e_{4}\right]=e_{3}$} \\
\hline $\mathrm{e}_{1,5}^{-}$ & {$\left[e_{1}, e_{4}\right]=\left[e_{2}, e_{3}\right]=-e_{1},\left[e_{2}, e_{4}\right]=-2 e_{2},\left[e_{3}, e_{4}\right]=e_{3}$} \\
\hline $\mathrm{e}_{1,6}$ & {$\left[e_{2}, e_{4}\right]=e_{2}$} \\
\hline $\mathrm{e}_{1,7}$ & {$\left[e_{1}, e_{4}\right]=e_{2}$} \\
\hline $\mathrm{e}_{1,8}$ & {$\left[e_{1}, e_{3}\right]=\left[e_{2}, e_{4}\right]=e_{2},\left[e_{1}, e_{4}\right]=e_{1}$} \\
\hline $\mathrm{e}_{1,9}$ & {$\left[e_{1}, e_{3}\right]=\left[e_{2}, e_{4}\right]=e_{2},\left[e_{1}, e_{4}\right]=\left[e_{2}, e_{3}\right]=e_{1}$} \\
\hline $\mathrm{e}_{1,10}$ & {$\left[e_{1}, e_{3}\right]=\left[e_{2}, e_{4}\right]=e_{2},\left[e_{1}, e_{4}\right]=e_{1},\left[e_{2}, e_{3}\right]=-e_{1}$} \\
\hline $\mathrm{e}_{2,1}$ & {$\left[e_{1}, e_{3}\right]=x e_{1},\left[e_{2}, e_{4}\right]=y e_{2}-e_{4}$} \\
\hline $\mathrm{e}_{2,2}$ & {$\left[e_{1}, e_{3}\right]=x e_{1},\left[e_{2}, e_{3}\right]=y e_{1},\left[e_{2}, e_{4}\right]=-e_{4}$} \\
\hline $\mathrm{e}_{2,3}$ & {$\left[e_{1}, e_{3}\right]=x e_{1},\left[e_{2}, e_{3}\right]=y e_{1},\left[e_{2}, e_{4}\right]=x e_{1}-e_{4},\left[e_{3}, e_{4}\right]=-x e_{4}$} \\
\hline$e_{3,1}$ & {$\left[e_{1}, e_{3}\right]=x e_{1},\left[e_{2}, e_{3}\right]=y e_{1}+z e_{2}-e_{4},\left[e_{3}, e_{4}\right]=z e_{4}$} \\
\hline $\mathrm{e}_{3,2}$ & {$\left[e_{1}, e_{3}\right]=\left[e_{2}, e_{4}\right]=x e_{1},\left[e_{2}, e_{3}\right]=y e_{1}+z e_{2}-e_{4},\left[e_{3}, e_{4}\right]=(z-x) e_{4}$} \\
\hline $\mathrm{e}_{4,1}$ & {$\left[e_{1}, e_{3}\right]=\left[e_{2}, e_{4}\right]=x e_{1}-e_{4},\left[e_{2}, e_{3}\right]=y e_{1}+x e_{2}-e_{3}$} \\
\hline $\mathrm{e}_{4,2}$ & {$\left[e_{1}, e_{3}\right]=\left[e_{2}, e_{4}\right]=x e_{2}-e_{4},\left[e_{2}, e_{3}\right]=-e_{3},\left[e_{3}, e_{4}\right]=x e_{3}$} \\
\hline$\overline{\mathrm{C}_{5,1}^{+}}$ & {$\left[e_{1}, e_{3}\right]=\left[e_{2}, e_{4}\right]=x e_{1}+y e_{2}-e_{4},\left[e_{1}, e_{4}\right]=\left[e_{2}, e_{3}\right]=y e_{1}+x e_{2}-e_{3}$} \\
\hline $\mathrm{e}_{5,2}^{+}$ & {$\left[e_{1}, e_{3}\right]=\left[e_{2}, e_{4}\right]=x e_{2}-e_{4},\left[e_{1}, e_{4}\right]=\left[e_{2}, e_{3}\right]=-e_{3},\left[e_{3}, e_{4}\right]=x e_{3}$} \\
\hline $\mathrm{C}_{5,1}^{-}$ & {$\left[e_{1}, e_{3}\right]=\left[e_{2}, e_{4}\right]=x e_{1}+y e_{2}-e_{4},\left[e_{1}, e_{4}\right]=y e_{1}-x e_{2}+e_{3},\left[e_{2}, e_{3}\right]=-y e_{1}+x e_{2}-e_{3}$} \\
\hline $\mathrm{C}_{5,2}^{-}$ & {$\left[e_{1}, e_{3}\right]=\left[e_{2}, e_{4}\right]=x e_{2}-e_{4},\left[e_{1}, e_{4}\right]=e_{3},\left[e_{2}, e_{3}\right]=-e_{3},\left[e_{3}, e_{4}\right]=x e_{3}$} \\
\hline
\end{tabular}

Table 6: Isomorphisms from the Lie algebras obtained in Table 4 onto the Lie algebras in Table 1

\begin{tabular}{|c|c|c|c|}
\hline \multicolumn{2}{|c|}{ Source } & Isomorphism & Target \\
\hline $\mathcal{B}_{1, \alpha}^{1}$ & $|\alpha|<1$ & $f_{1}=e_{1}, f_{2}=-\frac{\chi}{2} e_{1}+e_{3}, f_{3}=e_{4}, f_{4}=e_{2}$ & $\mathfrak{r}_{4,-1,-\alpha}$ \\
\hline $\mathcal{B}_{1, \alpha}^{1}$ & $|\alpha|>1, \alpha \neq-2$ & $f_{1}=e_{4}, f_{2}=e_{1}, f_{3}=-\frac{x}{2} e_{1}+e_{3}, f_{4}=-\frac{1}{\alpha} e_{2}$ & $\mathfrak{r}_{4,-\frac{1}{|\alpha|}, \frac{1}{|\alpha|}}$ \\
\hline $\mathcal{B}_{1, \alpha}^{2}$ & $|\alpha|<1, \alpha \neq 0$ & $f_{1}=e_{1}, f_{2}=e_{3}, f_{3}=-\frac{\chi}{\alpha} e_{2}+e_{4}, f_{4}=e_{2}$ & $\mathfrak{r}_{4,-1,-\alpha}$ \\
\hline $\mathcal{B}_{1, \alpha}^{2}$ & $|\alpha|>1, \alpha \neq-2$ & $f_{1}=-\frac{\chi}{\alpha} e_{2}+e_{4}, f_{2}=e_{1}, f_{3}=e_{3}, f_{4}=-\frac{1}{\alpha} e_{2}$ & $\mathfrak{r}_{4,-\frac{1}{\mid \alpha}, \frac{1}{|\alpha|}}$ \\
\hline $\mathcal{B}_{1,-2}^{1}$ & $x y \neq 0$ & $f_{1}=e_{2}+\frac{1}{x} e_{4}, f_{2}=-\frac{y}{2} e_{1}+e_{3}, f_{3}=y e_{1}, f_{4}=e_{2}$ & $\mathfrak{d}_{4,2}$ \\
\hline $\mathcal{B}_{1,-2}^{1}$ & $y=0$ & $f_{1}=x e_{2}+e_{4}, f_{2}=e_{3}, f_{3}=e_{1}, f_{4}=\frac{1}{2} e_{2}$ & $\mathfrak{r}_{4,-\frac{1}{2}, \frac{1}{2}}$ \\
\hline $\mathcal{B}_{1,-2}^{1}$ & $x=0$ & $f_{1}=e_{4}, f_{2}=-\frac{y}{2} e_{1}+e_{3}, f_{3}=e_{1}, f_{4}=\frac{1}{2} e_{2}$ & $\mathfrak{r}_{4,-\frac{1}{2}, \frac{1}{2}}$ \\
\hline $\mathcal{B}_{1,-2}^{2}$ & $x \neq 0$ & $f_{1}=e_{4}, f_{2}=\frac{1}{2 x} e_{1}+e_{2}+\frac{1}{x} e_{3}, f_{3}=-x e_{1}, f_{4}=-\frac{1}{x} e_{3}+\left(\frac{y+1}{x^{2}}\right) e_{4}$ & $\mathfrak{d}_{4,2}$ \\
\hline $\mathcal{B}_{1,-2}^{2}$ & $x=0$ & $f_{1}=e_{4}, f_{2}=-\frac{y}{2} e_{1}+e_{3}, f_{3}=e_{1}, f_{4}=\frac{1}{2} e_{2}$ & $\mathfrak{r}_{4,-\frac{1}{2}, \frac{1}{2}}$ \\
\hline $\mathcal{B}_{1,-1}^{1}$ & & $f_{1}=-\frac{1}{2} y e_{1}-x e_{2}+e_{3}, f_{2}=e_{1}, f_{3}=e_{4}, f_{4}=-e_{2}$ & $\mathfrak{r}_{4,-1,-1}$ \\
\hline $\mathcal{B}_{1,-1}^{2}$ & & $f_{1}=e_{3}, f_{2}=e_{1}, f_{3}=x e_{2}+e_{4}, f_{4}=-e_{2}$ & $\mathfrak{r}_{4,-1,-1}$ \\
\hline $\mathcal{B}_{1,0}^{1}$ & & $f_{1}=e_{2}, f_{2}=e_{1}, f_{3}=e_{3}, f_{4}=x e_{2}+e_{4}$ & $\mathfrak{r r}_{3,-1}$ \\
\hline $\mathcal{B}_{1,0}^{2}$ & & $f_{1}=-e_{2}, f_{2}=-\frac{\chi}{2} e_{1}+e_{3}, f_{3}=e_{1}, f_{4}=e_{4}$ & $\mathfrak{r r}_{3,-1}$ \\
\hline $\mathcal{B}_{1,1}^{1}$ & $x \neq 0$ & $f_{1}=e_{1}, f_{2}=x e_{3}-\frac{y}{2} e_{4}, f_{3}=\frac{y}{2} e_{1}+x e_{2}-e_{4}, f_{4}=e_{2}$ & $\mathfrak{r}_{4,-1,-1}$ \\
\hline $\mathcal{B}_{1,1}^{2}$ & & $f_{1}=e_{1}, f_{2}=-\frac{\chi}{2} e_{1}+e_{3}, f_{3}=e_{4}, f_{4}=e_{2}$ & $\mathfrak{r}_{4,-1,-1}$ \\
\hline $\mathcal{B}_{2}$ & & $f_{1}=e_{1}, f_{2}=-e_{4}, f_{3}=-\frac{x}{2} e_{1}+e_{3}, f_{4}=e_{2}$ & $\mathfrak{r}_{4,-1}$ \\
\hline $\mathcal{B}_{3, \alpha}^{1}$ & $\frac{1}{\alpha}>\frac{1}{2}$ & $f_{1}=e_{1}, f_{2}=-\chi e_{1}+\frac{\alpha-2}{\alpha} e_{3}, f_{3}=-\frac{\alpha-2}{\alpha} e_{4}, f_{4}=-e_{2}$ & $\mathfrak{d}_{4, \frac{1}{\alpha}}$ \\
\hline $\mathcal{B}_{3, \alpha}^{1}$ & $\frac{1}{\alpha}<\frac{1}{2}$ & $f_{1}=-\chi e_{1}+\frac{\alpha-2}{\alpha} e_{3}, f_{2}=e_{1}, f_{3}=\frac{\alpha-2}{\alpha} e_{4}, f_{4}=-e_{2}$ & $\mathfrak{d}_{4, \frac{\alpha-1}{\alpha}}$ \\
\hline
\end{tabular}




\begin{tabular}{|c|c|c|c|}
\hline $\mathcal{B}_{3,2}^{1}$ & $x \neq 0$ & $f_{1}=-x e_{1}, f_{2}=e_{3}, f_{3}=x e_{4}, f_{4}=-e_{2}$ & $\mathfrak{h}_{4}$ \\
\hline $\mathcal{B}_{3,2}^{1}$ & $x=0$ & $f_{1}=e_{3}, f_{2}=e_{1}, f_{3}=e_{4}, f_{4}=-e_{2}$ & $\mathfrak{d}_{4, \frac{1}{2}}$ \\
\hline $\mathcal{B}_{3, \alpha}^{2}$ & $\frac{1}{\alpha}>\frac{1}{2}$ & $f_{1}=e_{1}+x \alpha e_{2}-e_{4}, f_{2}=e_{3}, f_{3}=x \alpha e_{2}-e_{4}, f_{4}=-e_{2}+\frac{\alpha-1}{\alpha} e_{3}$ & $\mathfrak{d}_{4, \frac{1}{\alpha}}$ \\
\hline $\mathcal{B}_{3, \alpha}^{2}$ & $\frac{1}{\alpha}<\frac{1}{2}$ & $f_{1}=-x \alpha e_{2}+e_{3}+e_{4}, f_{2}=e_{1}, f_{3}=-x \alpha e_{2}+e_{4}, f_{4}=\frac{1}{\alpha} e_{1}-e_{2}$ & $\mathfrak{d}_{4, \frac{\alpha-1}{\alpha}}$ \\
\hline $\mathcal{B}_{3,2}^{2}$ & & $f_{1}=e_{3}, f_{2}=e_{1}, f_{3}=-2 x e_{2}+e_{4}, f_{4}=-e_{2}$ & $\mathfrak{d}_{4, \frac{1}{2}}$ \\
\hline $\mathcal{B}_{3, \frac{1}{2}}^{1}$ & & $f_{1}=e_{1}, f_{2}=\frac{1}{3} y e_{1}-\frac{1}{2} x e_{2}+e_{3}, f_{3}=-e_{4}, f_{4}=-e_{2}$ & $\mathfrak{d}_{4,2}$ \\
\hline $\mathcal{B}_{3, \frac{1}{2}}^{2}$ & $y \neq 0$ & $f_{1}=e_{1}, f_{2}=-e_{3}-\frac{x}{y} e_{4}, f_{3}=x e_{1}-\frac{1}{2} y e_{2}+e_{4}, f_{4}=-\frac{2}{y} e_{4}$ & $\mathfrak{d}_{4,2}$ \\
\hline $\mathcal{B}_{3,1}$ & $x=0, y=0$ & $f_{1}=e_{1}, f_{2}=z e_{1}+e_{3}, f_{3}=-e_{4}, f_{4}=-e_{2}$ & $\mathfrak{d}_{4,1}$ \\
\hline $\mathcal{B}_{3,1}$ & $x \neq 0, y=0$ & $f_{1}=\frac{z}{x} e_{1}-e_{2}+\frac{1}{x} e_{3}, f_{2}=e_{4}, f_{3}=-\frac{z}{x} e_{1}-\frac{1}{x} e_{3}, f_{4}=e_{1}-\frac{1}{x} e_{4}$ & $\mathfrak{r}_{2} \mathfrak{r}_{2}$ \\
\hline $\mathcal{B}_{3,1}$ & $x=0, y z>0$ & $\begin{array}{l}f_{1}=-\frac{\sqrt{y z}}{2 y} e_{1}-\frac{1}{2} e_{2}-\frac{1}{2 \sqrt{y z}} e_{3}, f_{2}=-\sqrt{y z} e_{1}-y e_{2}+e_{4}, f_{3}=\frac{\sqrt{y z}}{2 y} e_{1}- \\
\frac{1}{2} e_{2}+\frac{1}{2 \sqrt{y z}} e_{3}, f_{4}=\sqrt{y z} e_{1}-y e_{2}+e_{4}\end{array}$ & $\mathfrak{r}_{2} \mathfrak{r}_{2}$ \\
\hline $\mathcal{B}_{3,1}$ & $x=0, y z<0$ & $f_{1}=-\frac{1}{y} e_{4}, f_{2}=\frac{1}{\sqrt{-y z}} e_{3}, f_{3}=\sqrt{-y z} e_{1}, f_{4}=-y e_{2}+e_{4}$ & $\mathfrak{r}_{2}^{\prime}$ \\
\hline $\mathcal{B}_{3,1}$ & $x=0, y \neq 0, z=0$ & $f_{1}=e_{1}, f_{2}=e_{3}, f_{3}=y e_{2}-e_{4}, f_{4}=-e_{2}$ & $\mathfrak{d}_{4,1}$ \\
\hline $\mathcal{B}_{3,1}$ & $x y \neq 0, x^{2}+4 y z=0$ & $\begin{array}{l}f_{1}=-y e_{2}+e_{4}, f_{2}=-\frac{x^{2}}{4 y} e_{1}-\frac{x}{2} e_{2}+e_{3}, f_{3}=\frac{x^{2}}{4} e_{1}+\frac{x y}{2} e_{2}-\frac{x}{2} e_{4}, f_{4}= \\
-e_{2}\end{array}$ & $\mathfrak{d}_{4,1}$ \\
\hline $\mathcal{B}_{3,1}$ & $x y \neq 0, x^{2}+4 y z>0$ & $\begin{array}{l}f_{1}=\frac{z}{\sqrt{x^{2}+4 y z}} e_{1}-\frac{x+\sqrt{x^{2}+4 y z}}{2 \sqrt{x^{2}+4 y z}} e_{2}+\frac{1}{\sqrt{x^{2}+4 y z}} e_{3}, f_{2}=\left(-x+\sqrt{x^{2}+4 y z}\right) e_{1}- \\
2 y e_{2}+2 e_{4}, f_{3}=-\frac{z}{\sqrt{x^{2}+4 y z}} e_{1}+\frac{x-\sqrt{x^{2}+4 y z}}{2 \sqrt{x^{2}+4 y z}} e_{2}-\frac{1}{\sqrt{x^{2}+4 y z}} e_{3}, f_{4}= \\
-\frac{x+\sqrt{x^{2}+4 y z}}{2} e_{1}-y e_{2}+e_{4}\end{array}$ & $\mathfrak{r}_{2} \mathfrak{r}_{2}$ \\
\hline $\mathcal{B}_{3,1}$ & $x y \neq 0, x^{2}+4 y z<0$ & $\begin{array}{l}f_{1}=\frac{\sqrt{-x^{2}-4 y z}}{2} e_{1}-e_{2}, f_{2}=-\frac{x \sqrt{-x^{2}-4 y z}-4 z}{2 \sqrt{-x^{2}-4 y z}} e_{1}-\frac{y \sqrt{-x^{2}-4 y z}+x}{\sqrt{-x^{2}-4 y z}} e_{2}+ \\
\frac{2}{\sqrt{-x^{2}-4 y z}} e_{3}+e_{4}, f_{3}=\frac{\sqrt{-x^{2}-4 y z}}{2} e_{1}, f_{4}=-\frac{x}{2} e_{1}-y e_{2}+e_{4}\end{array}$ & $\mathfrak{r}_{2}^{\prime}$ \\
\hline $\mathcal{B}_{4}$ & $x=0$ & $f_{1}=-e_{1}, f_{2}=y e_{1}+e_{3}-e_{4}, f_{3}=e_{4}, f_{4}=-e_{2}$ & $\mathfrak{d}_{4,1}$ \\
\hline $\mathcal{B}_{4}$ & $x \neq 0$ & $f_{1}=\frac{x-y}{x} e_{1}-\frac{1}{x} e_{3}, f_{2}=-x e_{1}+e_{4}, f_{3}=\frac{y}{x} e_{1}-e_{2}+\frac{1}{x} e_{3}, f_{4}=e_{4}$ & $\mathfrak{r}_{2} \mathfrak{r}_{2}$ \\
\hline $\mathcal{B}_{5,1}^{+}$ & & $f_{1}=-\chi e_{2}-e_{3}+e_{4}, f_{2}=e_{1}, f_{3}=e_{3}, f_{4}=e_{1}+e_{2}$ & $\mathfrak{d}_{4,2}$ \\
\hline $\mathcal{B}_{5,2}^{+}$ & $x \neq 0$ & $f_{1}=\frac{1}{2} e_{2}-\frac{2}{x} e_{4}, f_{2}=e_{3}, f_{3}=\frac{x}{2} e_{1}+e_{3}, f_{4}=\frac{4}{x} e_{4}$ & $\mathfrak{d}_{4,2}$ \\
\hline $\mathcal{B}_{5,2}^{+}$ & $x=0$ & $f_{1}=e_{3}+e_{4}, f_{2}=e_{1}, f_{3}=e_{3}, f_{4}=-e_{1}+e_{2}$ & $\mathfrak{d}_{4,2}$ \\
\hline $\mathcal{B}_{5,3}^{+}$ & $x \neq 0$ & $f_{1}=-e_{3}-e_{4}, f_{2}=e_{1}, f_{3}=x e_{1}+x e_{2}+e_{3}, f_{4}=-\frac{1}{x} e_{3}$ & $\mathfrak{d}_{4,2}$ \\
\hline $\mathcal{B}_{5,3}^{+}$ & $x=0$ & $f_{1}=e_{4}, f_{2}=e_{1}, f_{3}=e_{3}, f_{4}=e_{2}$ & $\mathfrak{d}_{4,2}$ \\
\hline $\mathcal{B}_{5,4}^{+}$ & $x \neq 0$ & $f_{1}=e_{3}+e_{4}, f_{2}=e_{1}, f_{3}=-x e_{1}+x e_{2}+e_{3}, f_{4}=-e_{1}+e_{2}$ & $\mathfrak{d}_{4,2}$ \\
\hline $\mathcal{B}_{5,4}^{+}$ & $x=0$ & $f_{1}=-e_{3}+e_{4}, f_{2}=e_{1}, f_{3}=e_{3}, f_{4}=e_{1}+e_{2}$ & $\mathfrak{d}_{4,2}$ \\
\hline $\mathcal{B}_{5,1}^{-}$ & & $f_{1}=-x e_{2}+e_{4}, f_{2}=e_{1}, f_{3}=-e_{3}, f_{4}=e_{2}$ & $\mathfrak{d}_{4,2}$ \\
\hline $\mathcal{B}_{5,2}^{-}$ & $x \neq 0$ & $f_{1}=e_{2}+\frac{4}{x} e_{4}, f_{2}=e_{3}, f_{3}=x e_{1}+2 e_{3}, f_{4}=-\frac{4}{x} e_{4}$ & $\mathfrak{d}_{4,2}$ \\
\hline $\mathcal{B}_{5,2}^{-}$ & $x=0$ & $f_{1}=e_{3}+e_{4}, f_{2}=e_{1}, f_{3}=-e_{3}, f_{4}=e_{1}+e_{2}$ & $\mathfrak{d}_{4,2}$ \\
\hline
\end{tabular}

Table 7: Isomorphisms from the Lie algebras obtained in Table 5 onto the Lie algebras in Table 1

\begin{tabular}{|ll|l|c|}
\hline Source & \multicolumn{1}{|c|}{ Isomorphism } & Target \\
\hline $\mathcal{C}_{1,1} \quad-1 \leq \alpha<1$ & $f_{1}=e_{1}, f_{2}=e_{3}, f_{3}=e_{2}, f_{4}=-e_{4}$ & $\mathfrak{r}_{4,-1, \alpha}$ \\
\hline $\mathcal{C}_{1,1} \quad \alpha<-1$ & $f_{1}=e_{2}, f_{2}=e_{1}, f_{3}=e_{3}, f_{4}=-\frac{1}{\alpha} e_{4}$ & $\mathfrak{r}_{4, \frac{1}{\alpha},-\frac{1}{\alpha}}$ \\
\hline $\mathcal{C}_{1,1} \quad \alpha>1$ & $f_{1}=e_{2}, f_{2}=e_{3}, f_{3}=e_{1}, f_{4}=-\frac{1}{\alpha} e_{4}$ & $\mathfrak{r}_{4,-\frac{1}{\alpha}, \frac{1}{\alpha}}$ \\
\hline $\mathcal{C}_{1,1} \quad \alpha=1$ & $f_{1}=e_{3}, f_{2}=e_{1}, f_{3}=e_{2}, f_{4}=e_{4}$ & $\mathfrak{r}_{4,-1,-1}$ \\
\hline $\mathcal{C}_{1,2}$ & $f_{1}=e_{3}, f_{2}=-e_{2}, f_{3}=e_{1}, f_{4}=e_{4}$ & $\mathfrak{r}_{4,-1}$ \\
\hline $\mathcal{C}_{1,3} \quad 0<\alpha \leq 2$ & $f_{1}=e_{2}-e_{3}, f_{2}=e_{1}, f_{3}=e_{2}, f_{4}=\frac{\alpha-1}{\alpha} e_{1}-e_{4}$ & $\mathfrak{d}_{4, \frac{1}{\alpha}}$ \\
\hline $\mathcal{C}_{1,3} \quad \alpha<0$ or $\alpha>2$ & $f_{1}=e_{1}+\alpha e_{2}, f_{2}=e_{3}, f_{3}=e_{2}, f_{4}=e_{3}-e_{4}$ & $\mathfrak{d}_{4, \frac{\alpha-1}{\alpha}}$ \\
\hline
\end{tabular}




\begin{tabular}{|c|c|c|c|}
\hline $\mathrm{C}_{1,4}$ & & $f_{1}=e_{3}, f_{2}=e_{1}-e_{2}, f_{3}=-e_{2}, f_{4}=-e_{4}$ & $\mathfrak{d}_{4,1}$ \\
\hline $\mathrm{C}_{1,5}^{+}$ & & $f_{1}=e_{2}, f_{2}=e_{3}, f_{3}=e_{1}, f_{4}=e_{4}$ & $\mathfrak{d}_{4,2}$ \\
\hline $\mathrm{C}_{1,5}^{-}$ & & $f_{1}=e_{2}, f_{2}=-e_{3}, f_{3}=e_{1}, f_{4}=e_{4}$ & $\mathfrak{d}_{4,2}$ \\
\hline $\mathrm{e}_{1,6}$ & & $f_{1}=-e_{4}, f_{2}=e_{2}, f_{3}=e_{3}, f_{4}=e_{1}$ & $\mathfrak{r r}_{3,0}$ \\
\hline $\mathcal{C}_{1,7}$ & & $f_{1}=e_{1}, f_{2}=e_{4}, f_{3}=e_{2}, f_{4}=e_{3}$ & $\mathfrak{r h}_{3}$ \\
\hline $\mathrm{e}_{1,8}$ & & $f_{1}=e_{1}, f_{2}=e_{3}, f_{3}=e_{2}, f_{4}=-e_{4}$ & $\mathfrak{d}_{4,1}$ \\
\hline $\mathrm{C}_{1,9}$ & & $f_{1}=\frac{1}{2} e_{3}-\frac{1}{2} e_{4}, f_{2}=e_{1}-e_{2}, f_{3}=-\frac{1}{2} e_{3}-\frac{1}{2} e_{4}, f_{4}=e_{1}+e_{2}$ & $\mathfrak{r}_{2} \mathfrak{r}_{2}$ \\
\hline $\mathcal{C}_{1,10}$ & & $f_{1}=-e_{4}, f_{2}=e_{3}, f_{3}=e_{1}, f_{4}=-e_{2}$ & $\mathfrak{r}_{2}^{\prime}$ \\
\hline $\mathrm{e}_{2,1}$ & $x=0$ & $f_{1}=-e_{2}, f_{2}=-y e_{2}+e_{4}, f_{3}=e_{1}, f_{4}=e_{3}$ & $\mathfrak{r r}_{3,0}$ \\
\hline $\mathrm{C}_{2,1}$ & $x \neq 0$ & $f_{1}=-\frac{1}{x} e_{3}, f_{2}=e_{1}, f_{3}=-e_{2}, f_{4}=-x y e_{2}+x e_{4}$ & $\mathfrak{r}_{2} \mathfrak{r}_{2}$ \\
\hline $\mathrm{C}_{2,2}$ & $x \neq 0$ & $f_{1}=-\frac{1}{x} e_{3}, f_{2}=e_{1}, f_{3}=\frac{y}{x} e_{1}-e_{2}, f_{4}=e_{4}$ & $\mathfrak{r}_{2} \mathfrak{r}_{2}$ \\
\hline $\mathrm{C}_{2,2}$ & $x=0, y \neq 0$ & $f_{1}=e_{4}, f_{2}=-y e_{1}, f_{3}=e_{3}, f_{4}=-e_{2}$ & $\mathfrak{r}_{4,0}$ \\
\hline $\mathrm{C}_{2,2}$ & $x=0, y=0$ & $f_{1}=-e_{2}, f_{2}=e_{4}, f_{3}=e_{1}, f_{4}=e_{3}$ & $\mathfrak{r r}_{3,0}$ \\
\hline $\mathrm{C}_{2,3}$ & $x \neq 0$ & $f_{1}=\frac{y}{x} e_{1}-e_{2}, f_{2}=-x e_{1}+e_{4}, f_{3}=e_{2}-\frac{1}{x} e_{3}, f_{4}=e_{1}$ & $\mathfrak{r}_{2} \mathfrak{r}_{2}$ \\
\hline $\mathrm{C}_{2,3}$ & $x=0, y \neq 0$ & $f_{1}=e_{4}, f_{2}=-y e_{1}, f_{3}=e_{3}, f_{4}=-e_{2}$ & $\mathfrak{r}_{4,0}$ \\
\hline $\mathcal{C}_{2,3}$ & $x=0, y=0$ & $f_{1}=e_{1}-e_{2}, f_{2}=e_{4}, f_{3}=e_{1}, f_{4}=e_{3}$ & $\mathfrak{r r}_{3,0}$ \\
\hline $\mathrm{e}_{3,1}$ & $z=0, x \neq 0$ & $f_{1}=e_{1}, f_{2}=-\frac{1}{x} e_{4}, f_{3}=-\frac{y}{x} e_{1}+e_{2}, f_{4}=-\frac{1}{x} e_{3}$ & $\mathfrak{r}_{4,0}$ \\
\hline $\mathrm{e}_{3,1}$ & $z=0, x=0$ & $f_{1}=-e_{2}, f_{2}=e_{2}-e_{3}, f_{3}=y e_{1}-e_{4}, f_{4}=e_{1}$ & $\mathfrak{r h}_{3}$ \\
\hline $\mathrm{e}_{3,1}$ & $z \neq 0, x=0$ & $f_{1}=\frac{1}{z} e_{3}, f_{2}=e_{4}, f_{3}=-2 y e_{1}-2 z e_{2}+e_{4}, f_{4}=e_{1}$ & $\mathfrak{r r}_{3,-1}$ \\
\hline $\mathrm{C}_{3,1}$ & $z \neq 0,-1<\frac{-x}{z}<1$ & $f_{1}=e_{4}, f_{2}=\frac{2 y z}{x-z} e_{1}-2 z e_{2}+e_{4}, f_{3}=e_{1}, f_{4}=\frac{1}{z} e_{3}$ & $\mathfrak{r}_{4,-1,-\frac{x}{z}}$ \\
\hline $\mathrm{C}_{3,1}$ & $z \neq 0, x \neq 0, \frac{-x}{z}>1$ & $f_{1}=e_{1}, f_{2}=\frac{2 y z}{x-z} e_{1}-2 z e_{2}+e_{4}, f_{3}=e_{4}, f_{4}=-\frac{1}{x} e_{3}$ & $\mathfrak{r}_{4, \frac{z}{x},-\frac{z}{x}}$ \\
\hline $\mathcal{C}_{3,1}$ & $z \neq 0, x \neq 0, \frac{-x}{z}<-1$ & $f_{1}=e_{1}, f_{2}=e_{4}, f_{3}=\frac{2 y z}{x-z} e_{1}-2 z e_{2}+e_{4}, f_{4}=-\frac{1}{x} e_{3}$ & $\mathfrak{r}_{4,-\frac{z}{x}, \frac{z}{x}}$ \\
\hline$e_{3,1}$ & $z \neq 0, \frac{-X}{z}=1$ & $f_{1}=-\frac{y}{2 x} e_{1}+e_{2}+\frac{1}{2 x} e_{4}, f_{2}=e_{4}, f_{3}=e_{1}, f_{4}=\frac{1}{x} e_{3}$ & $\mathfrak{r}_{4,-1,-1}$ \\
\hline $\mathrm{C}_{3,1}$ & $z \neq 0, y \neq 0, x=z$ & $f_{1}=e_{4}, f_{2}=-\frac{y}{z} e_{1}, f_{3}=e_{2}-\frac{1}{2 z} e_{4}, f_{4}=\frac{1}{z} e_{3}$ & $\mathfrak{r}_{4,-1}$ \\
\hline$e_{3,1}$ & $z \neq 0, y=0, x=z$ & $f_{1}=e_{4}, f_{2}=-2 z e_{2}+e_{4}, f_{3}=e_{1}, f_{4}=\frac{1}{z} e_{3}$ & $\mathfrak{r}_{4,-1,-1}$ \\
\hline $\mathrm{e}_{3,2}$ & $z=0, x=0$ & $f_{1}=-e_{2}, f_{2}=e_{2}-e_{3}, f_{3}=y e_{1}-e_{4}, f_{4}=e_{1}$ & $\mathfrak{r h}_{3}$ \\
\hline $\mathrm{C}_{3,2}$ & $z=0, x \neq 0$ & $f_{1}=e_{4}, f_{2}=-\frac{y}{x} e_{1}+e_{2}+\frac{1}{x} e_{4}, f_{3}=-x e_{1} f_{4}=-\frac{1}{x} e_{3}$ & $\mathfrak{d}_{4,1}$ \\
\hline $\mathrm{e}_{3,2}$ & $z \neq 0, x=0$ & $f_{1}=\frac{1}{z} e_{3}, f_{2}=e_{4}, f_{3}=-2 y e_{1}-2 z e_{2}+e_{4} f_{4}=e_{1}$ & $\mathfrak{r r}_{3,-1}$ \\
\hline $\mathrm{C}_{3,2}$ & $z \neq 0, x \neq 0, \frac{z}{x}=\frac{1}{2}$ & $f_{1}=-\frac{1}{2 z} e_{4}, f_{2}=-\frac{y}{z} e_{1}+e_{2}, f_{3}=e_{1} f_{4}=-\frac{1}{2 z} e_{3}$ & $\mathfrak{h}_{4}$ \\
\hline$e_{3,2}$ & $z \neq 0, x \neq 0, \frac{z}{x}<\frac{1}{2}$ & $\begin{array}{l}f_{1}=\frac{y(x-2 z)}{z} e_{1}+e_{4}, f_{2}=(x-2 z) e_{2}+e_{4}, f_{3}=-x(x- \\
2 z) e_{1}, f_{4}=-\frac{y(x-2 z)}{x^{2}} e_{2}-\frac{1}{x} e_{3}\end{array}$ & $\mathfrak{d}_{4, \frac{x-z}{x}}$ \\
\hline$e_{3,2}$ & $z \neq 0, x \neq 0, \frac{z}{x}>\frac{1}{2}$ & $\begin{array}{l}f_{1}=(x-2 z) e_{2}+e_{4}, f_{2}=\frac{y(x-2 z)}{z} e_{1}+e_{4}, f_{3}=x(x- \\
2 z) e_{1}, f_{4}=-\frac{y(x-2 z)}{x^{2}} e_{2}-\frac{1}{x} e_{3}\end{array}$ & $\mathfrak{d}_{4, \frac{z}{x}}$ \\
\hline $\mathrm{e}_{4,1}$ & & $\begin{array}{l}f_{1}=-y e_{1}-x e_{2}+e_{3}, f_{2}=e_{1}, f_{3}=-x e_{1}+e_{4} f_{4}= \\
-x e_{1}-e_{2}+e_{4}\end{array}$ & $\mathfrak{d}_{4,1}$ \\
\hline $\mathrm{C}_{4,2}$ & & $f_{1}=e_{3}, f_{2}=e_{1}, f_{3}=-x e_{2}+e_{4} f_{4}=-e_{2}$ & $\mathfrak{d}_{4,1}$ \\
\hline $\mathcal{C}_{5,1}^{+}$ & & $\begin{array}{l}f_{1}=\frac{1}{2} e_{1}-\frac{1}{2} e_{2}, f_{2}=(-x+y) e_{1}+(x-y) e_{2}-e_{3}+e_{4}, f_{3}= \\
-\left(\frac{1}{2}+x+y\right) e_{1}-\left(\frac{1}{2}+x+y\right) e_{2}+e_{3}+e_{4}, f_{4}=-(x+y) e_{1}- \\
(x+y) e_{2}+e_{3}+e_{4}\end{array}$ & $\mathfrak{r}_{2} \mathfrak{r}_{2}$ \\
\hline $\mathrm{C}_{5,2}^{+}$ & & $\begin{array}{l}f_{1}=\frac{1}{2} e_{1}-\frac{1}{2} e_{2}, f_{2}=-x e_{2}-e_{3}+e_{4}, f_{3}=-\frac{1}{2} e_{1}-\frac{1}{2} e_{2}, f_{4}= \\
-x e_{2}+e_{3}+e_{4}\end{array}$ & $\mathfrak{r}_{2} \mathfrak{r}_{2}$ \\
\hline $\mathrm{C}_{5,1}^{-}$ & & $\begin{array}{l}f_{1}=y e_{1}-(x+1) e_{2}+e_{3}, f_{2}=-(x+1) e_{1}-y e_{2}+e_{4}, f_{3}= \\
y e_{1}-x e_{2}+e_{3} f_{4}=-x e_{1}-y e_{2}+e_{4}\end{array}$ & $\mathfrak{r}_{2}^{\prime}$ \\
\hline $\mathrm{e}_{5,2}^{-}$ & & $f_{1}=-e_{2}-e_{3}, f_{2}=e_{1}-x e_{2}+e_{4}, f_{3}=-e_{3} f_{4}=-x e_{2}+e_{4}$ & $\mathfrak{r}_{2}$ \\
\hline
\end{tabular}


Acknowledgments: The authors would like to thank sincerely Professor Mohamed Boucetta for his many suggestions which were of great help. The authors would like to thank also the reviewer for his effort to improve the article.

Conflict of interest: Authors state no conflict of interest.

\section{References}

[1] D. V. Alekseevsky, C. Medori and A. Tomassini, Homogeneous para-Kähler Einstein manifolds, Russian Mathematical Surveys, Volume 64, Number 1

[2] C. Bai, Left-symmetric bialgebras and an analogue of the classical Yang-Baxter equation, Commun. Contemp. Math. 10 (2008), no. 2, 221-260.

[3] S. Benayadi, M. Boucetta, On para-Kähler and hyper-para-Kähler Lie algebras, J. Algebra 436, (2015),61-101.

[4] O. Bouzour and M. W. Mansouri, Bi-Lagrangian Structure on the Symplectic Affine Lie Algebra aff (2, $\mathbb{R})$ Journal of Geometry and Symmetry in Physics-56, (2020), 45-57.

[5] N. B. Boyom, Métriques Kählériennes affinement plates de certaines variétés symplectiques I Proc. London Math. Soc. (1993), 358-80.

[6] G. Calvaruso, A complete classification of four-dimensional para-Kähler Lie algebras, Complex Manifolds (2), (2015), 1-10.

[7] F. Etayo, R. Santamaria and U. R. Trias The geometry of a bi-Lagrangian manifold, Dif Geo and its App (24), (1), (2006), 33-59.

[8] M. J. D. Hamilton, Bi-Lagrangian structures on nilmanifolds, Jour of Geom and Phys (140), (2019), 10-25. arXiv:1810.06518.

[9] H. Hess, Connexions on symplectic manifolds and geometric quantization, Differential geometrical methods in mathematical physics (Proc. Conf., Aix-en- Provence/Salamanca) (1979), pp 153-166.

[10] G. Ovando Four Dimensional Symplectic Lie Algebras, Contributions to Algebra and Geometry, 47 (2006), No. 2, $419-434$.

[11] A. Konyaev, Nijenhuis geometry II: Left-symmetric algebras and linearization problem for Nijenhuis operators, Diff. Geom. App., 74 (2021), 32 pp.

[12] N. K. Smolentsev and I. Y. Shagabudinova, On the classification of left-invariant para-Kähler structures on four-dimensional Lie groups arXiv:2008.05664 